\title{
Investigating the Transition between Polymer Melts and Solutions in Non- linear Elongational Flow
}

Alexis Andrél,3, Taisir Shahid,1,2, Filip Oosterlinck ${ }^{2}$, Christian Clasen ${ }^{3}$ *Evelyne van Ruymbeke ${ }^{1}$

1. Bio and Soft Matter, Institute on Condensed Matter and Nano-science, Université catholique de Louvain, Louvain-la-Neuve, Belgium.

2. DSM Materials Science Center, P.O. Box 18, NL-6160 MD Geleen, The Netherlands.

3. Department of Chemical Engineering, KU Leuven, Celestijnenlaan 200f, 3001 Leuven, Belgium.

*evelyne.vanruymbeke@uclouvain.be

For Table of Contents use only:

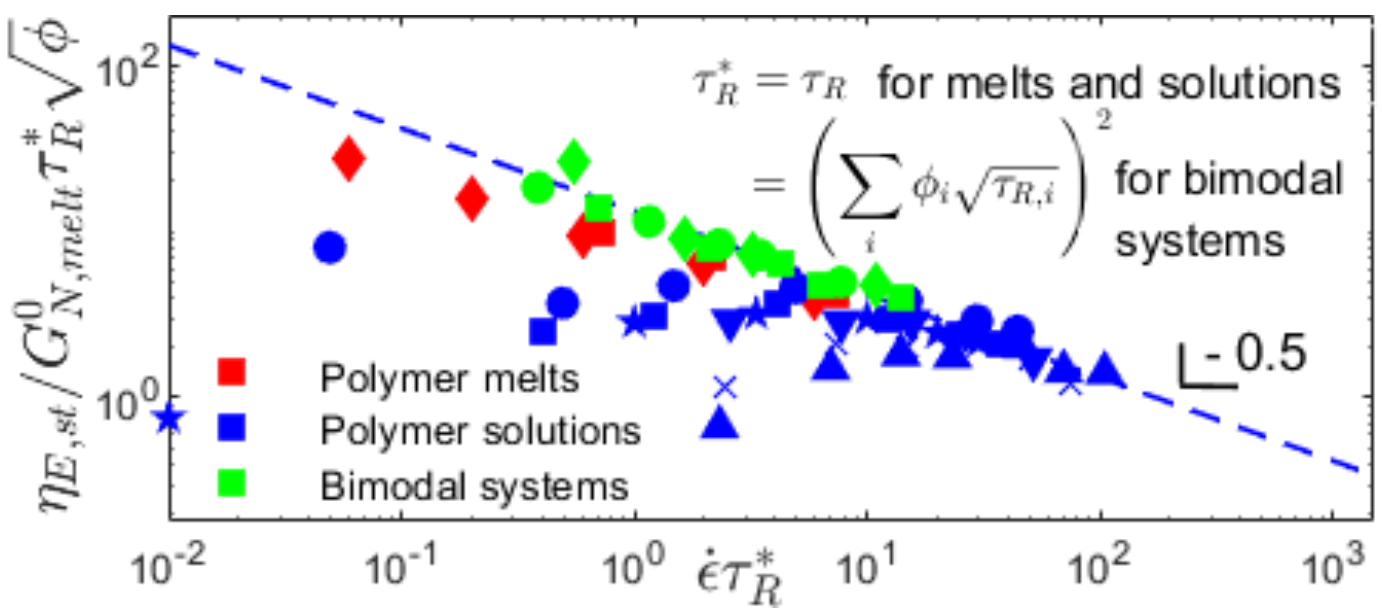




\begin{abstract}
The Doi-Edwards tube model, coupled with relaxation mechanisms such as reptation, contour length fluctuation and constraint release, allows to quantitatively predict the linear viscoelastic properties of entangled polymers. However, for non-linear elongational flows, large discrepancies between theoretical predictions based on the tube model and experimental results still persist today. This is in particular obvious for the experimentally observed strong qualitative differences in extensional flow of entangled polystyrene (PS) melts and solutions, despite having the same number of entanglements and exhibiting the same linear viscoelastic behaviour. The cause of this non-universality is often attributed either to a monomeric friction reduction, or to an interchain pressure effect.
\end{abstract}

In this work, we investigate the changes in extensional flow behavior going from polymer solutions to the melt state. For this purpose, we measure with a filament stretching rheometer the non-linear extensional responses of differently long PS chains, both in the melt state and diluted in short chain matrices of the same polymer at varying concentrations. These concentrations have been chosen sufficiently high, such that the chains stay entangled. This allows us to discuss the influence of concentration and molar mass on the steady state elongational viscosity to highlight scaling relations.

The purpose of the present work is to conduct well-defined experiments to further investigate how the steady extensional viscosity of polymer solutions and blends varies with the concentration and with the molecular weight of the chains 


\section{Introduction}

The Doi-Edwards tube model ${ }^{1}$, coupled with relaxation mechanisms such as reptation, Contour Length Fluctuation (CLF) and Thermal Constraint Release (TCR), allows to quantitatively predict the linear viscoelastic (LVE) properties of entangled polymeric systems. ${ }^{2}$ Specifically, the model allows to express the linear response as a universal function that only depends on the average number of entanglements $Z$ along the chains, the average molar mass between two entanglements $M_{e}$, and the entanglement relaxation time $\tau_{e}{ }^{3}$ These two latter are materialspecific parameters related to a segment between entanglements, which is the largest stresscarrying element of the chain.

The tube model constitutes the foundation of the so-called "standard molecular theory" 4 , which aims to predict the linear and the non-linear properties of entangled systems. In this theory, two additional mechanisms, namely Chain Stretch (CS) and Convective Constraint Release (CCR) are present and can become dominant when entering the non-linear flow regime. ${ }^{3}$ Despite this extension of the tube model in the non-linear regime, large discrepancies between theory and experimental results still persist today. In particular, experimental data show that polystyrene (PS) melts adopt a monotonous extension thinning behavior with a scaling of $\eta_{E} \propto M_{W} \dot{\varepsilon}^{-0.5}$, while PS solutions with the same $Z$ initially exhibit extensional thinning and, at elongation rates comparable to the reciprocal Rouse time $\tau_{R}^{-1}$, switch to an extension-thickening behavior. ${ }^{6-8}$ These experimental observations contradict the standard molecular theory, which predicts, for all entangled systems, an upturn of the extensional viscosity beyond $\dot{\varepsilon}=\tau_{R}^{-1}$ due to the rapid stretching of the chains. ${ }^{4}$ This unexpected difference of the qualitative behavior between polymer melts and solutions potentially suggests that additional mechanisms need to be added to the model to capture the correct molecular picture. 
The interchain pressure effect (IP) ${ }^{4}$, initially postulated by Marrucci and Ianniruberto, is the first proposed mechanism that allows to predict the experimentally observed scaling of polymer melts. In this model, the contraction of the tube radius due to its affine deformation is balanced by an entropic force exerted on the tube walls due to the confinement of the chain. This approach leads to the introduction of a new characteristic time, namely, the tube diameter relaxation time $\tau_{a}$, which scales with $\tau_{R}$. Based on this idea, Wagner et al. ${ }^{9}$ developed, in the framework of the molecular stress function theory, a full constitutive equation of which the predictions are in semi-quantitative agreement with the entire response of monodisperse PS melts. Subsequently, this Extended Interchain Pressure (EIP) model has been successfully applied to PS solutions in oligomeric styrene $(\mathrm{OS})^{10}$ and in diethyl phthalate (DEP). ${ }^{11}$ Nevertheless, the EIP model requires two stretch relaxation times; the Rouse time of the solutions and the Rouse time of the corresponding melt, which is questionable as a Rouse process should not depend on the concentration. ${ }^{12}$ In addition, it was also shown that, for binary blends, $\tau_{a}$ obtained by fitting the experimental data varies with the composition, contrary to what was expected from the model. ${ }^{13}$ Very recently, Narimissa et al. ${ }^{14}$ adapted the EIP model so that it relies upon one single stretch relaxation time. The main prediction of this rework is that polymer concentration and molar mass of the solvent (if equal or larger than a quarter of $M_{e}$ ) have no effect on the steady-state elongational viscosity $\eta_{E}$ (for a Rouse Weissenberg number, $\left.W i_{R}=\dot{\epsilon} \tau_{R}>>1\right)$. A quantitative agreement between theoretical and experimental data was obtained with elongational data on monodisperse PS melt, concentrated PS solutions in OS and PS diluted in DEP. However, the model still has to be applied to polymers of different nature.

On the other hand, Ianniruberto et al. ${ }^{15}$ and Yaoita et al. ${ }^{16}$ proposed that the non-universality of polymer melts and solutions is caused by Stretch-Orientation induced Reduction in Friction (SORF). ${ }^{17,18}$ Under fast elongational flow, the local environment of the chains, at the Kuhn 
segment scale, becomes strongly anisotropic, resulting in a decrease of the monomeric friction $\zeta_{0}$, and thus in a reduction of the overall level of stretch. As solvent molecules tend to stay isotropic, dilution tends to impede the reduction of friction. This hypothesis has been scrutinized and reinforced by molecular simulations done by Ianniruberto et al. ${ }^{19}$ and Masubuchi et al. ${ }^{20}$ Also, Desai and Larson ${ }^{21}$ succeeded to retrieve the monotonic extension thinning of the melt and the extension thickening of the solution by incorporating a friction coefficient $\zeta$, which depends on a stretch/orientation parameter, within the Doi-EdwardsMarrucci-Grizzuti (DEMG) model ${ }^{3}$.

However, they did not include in their model the work of Huang et al. ${ }^{22}$, which suggests that the friction depends also on the solvent. Huang et al. showed that solutions of PS in short chain PS/styrene oligomers (with molar masses for the short chains ranging from $1 \mathrm{k}$ to $4 \mathrm{k}$, and equal molar mass for the long chains) exhibit different extensional responses. They postulated that nematic interactions between the short molecules and the long polymeric chains would bring some anisotropy to the short molecules, and therefore cause a decrease of the monomeric friction. On this basis, Ianniruberto ${ }^{23}$ developed a multi-mode model, which could successfully fit the extensional properties of the PS solutions of Huang et al. ${ }^{24}$ Their model includes a nematic interaction parameter $\epsilon$, which is defined as a ratio of the order parameter of the solvent molecules to the order parameter of the long chains. This new, non-linear parameter was found to vary with the molar mass of the solvent molecules. ${ }^{25}$ It vanishes for the smallest solvent molecule investigated and, according to the data of Shahid at $a l .{ }^{26}$, remains constant above an oligomeric molecular weight of 9k for PS.

Recently, Matsumiya et al..$^{27}$ have investigated experimentally the mechanism of monomeric friction reduction through the extensional responses of two unentangled melts; a polystyrene of $27 \mathrm{k}$ and a poly(p-tert-butylstyrene) of $53 \mathrm{k}$. For both polymeric systems, the steady state 
extensional viscosity exhibited an extension-thickening behavior followed by extensionthinning with increasing strain rate. They attributed the thickening to the finite extensibility of the polymer chains and the thinning to the reduction of monomeric friction, which eventually overcomes the finite extensibility effect due to the high degree of orientation/stretch of the chains. They successfully modeled the steady state data by using a finite extensible nonlinear elasticity (FENE) bead-spring model and by fitting experimentally the $\zeta$ reduction. However, their model failed to reproduce the transient stress growth coefficient $\eta_{E}^{+}(t)$ and the relaxation data of the polymeric systems. They hypothesized that, on start-up and cessation of fast flows, the transient $\zeta$ reduction was delayed compared to the stress evolution. As a result, the $\zeta$ reduction would be fully characterized not only by the stretch and orientation but also by their rate of change. This idea has been further investigated by Ianniruberto et al. ${ }^{28}$ by means of Brownian simulations of Fraenkel chains. A rather good quantitative fit of the start-up data and most of the relaxation data has been obtained with their model thanks to the introduction of a time delay for the $\zeta$ reduction in the fastest flow.

Very recently, Bobbili and Milner showed, based on bead-spring simulations, that friction reduction occurs under large flow. ${ }^{29}$ However, in this approach, the role played by the molecular environment on the friction reduction is not clear, contrary to the conclusion of Ianniruberto et al. ${ }^{17,18}$. Furthermore, it should be mentioned that in this work, friction reduction is determined by looking at the chain diffusion in the flow-direction, which does not mean that the diffusion of the chain along its contour (i.e. in the three directions) is faster under large flow. This last condition would be required in order to consider friction reduction in specific models, as for example the ones recently introduced based on a Pincus blob picture. ${ }^{30}$

Today, it stays unclear if the idea of friction reduction can be extended to entangled polymeric systems. O’Connor et al. ${ }^{31}$ reported Kremer-Grest bead-spring molecular dynamics (MD) 
simulations of stress relaxation of entangled melts after fast elongational flow. Ianniruberto and Marrucci. ${ }^{32}$ recently showed that these simulations are in agreement with the $\zeta$ reduction.

The purpose of the present work is to conduct well-defined experiments to further investigate how the steady extensional viscosity of polymer solutions and blends varies with the concentration and with the molecular weight of the chains . To this end, we have designed and prepared a new set of data, where polymeric systems were obtained by dissolving moderate and relatively high molecular weight monodisperse PS in oligomeric styrene (OS) or moderate molecular weight PS at different weight fraction. The OS has been selected such that it is sufficiently short in order to act as solvent completely miscible with PS, as well as sufficiently long, so that the polymer-matrix interaction remains unchanged due to the size of the solvent molecules, following the work of Shahid et al. ${ }^{26}$ All the prepared polymeric systems are measured in both linear oscillatory shear and extensional flows.

\section{Materials and Experimental details}

\section{II.1. Materials and chromatography}

Polystyrene of varying molar mass was commercially obtained from Polymer Source, Inc. (Montreal, Canada). The materials were chosen to ensure a polydispersity index (PDI) as low as possible. The molar mass and PDI of the ingredients have been determined with sizeexclusion chromatography (SEC), as well as by field flow fractionation (FFF) coupled with multi-angle light scattering (MALS) for the highest molar mass (performed at BASF, Germany). The $T_{\mathrm{g}}$ of the melts has also been determined by differential scanning calorimetry (DSC). A standard Heat-Cool-Heat procedure was carried out with a heating rate of $10 \mathrm{~K} / \mathrm{min}$ under an inert atmosphere. The results, which were found to agree with the specifications received from Polymer Source, Inc., are summarized in Table 1.

Table 1. Specifications of the oligomers and polymers 


\begin{tabular}{|l|c|c|c|}
\hline Sample & $M_{w}[\mathrm{~kg} / \mathrm{mol}]$ & PDI & $T_{g}\left[{ }^{\circ} \mathrm{C}\right]$ \\
\hline PS820 & 820 & 1.02 & 106.6 \\
\hline PS389 & 390 & 1.08 & 106.6 \\
\hline PS261 & 260.5 & 1.05 & 106.6 \\
\hline PS256 & 256 & 1.09 & - \\
\hline OS9 & 8.8 & 1.1 & 94.6 \\
\hline
\end{tabular}

\section{II.2. Preparation of solutions and bidisperse blends}

Solutions of the polystyrenes PS820, PS389 or PS261 in the oligomer OS9 were made using a precipitation process following a procedure inspired by ref [24]. The purchased PS samples have also been used to prepare three bidisperse blends. Once the respective PS and OS have been weighted according to the targeted weight fraction, they are dissolved in tetrahydrofuran (THF) in a sealed container under gentle magnetic stirring overnight $(>12 \mathrm{~h})$ to yield concentrations from 10 to $15 \mathrm{mg} / \mathrm{ml}$. Subsequently, the PS/OS/THF solution is added drop by drop into 10 times the volume of methanol under continuous stirring. Once the solution is added to the methanol, the stirring is interrupted and the solution is covered. After 1 to 2 hours of rest in the covered container, the PS and OS have precipitated and settled down. The precipitate is filtered off and subsequently dried under vacuum $(\sim 0.1$ bar $)$ at $70{ }^{\circ} \mathrm{C}$ for 4 days to remove the residual solvent.

The composition of the prepared PS systems and their glass transition temperature $T_{\mathrm{g}}$, which was determined by DSC (in the same way as for the melts), is summarized in Table 2. The molar mass between two entanglements, $M_{e}$, and the number of entanglement segments in a chain, $Z$, has been determined based on $M_{e}=15 \mathrm{~kg} / \mathrm{mol}$ in the melt state. The blends composed of PS820 and OS9 have been already used in ref. [33]. 
Table 2. Key properties of the polystyrene systems

\begin{tabular}{|l|c|c|c|c|}
\hline & $\begin{array}{c}\text { Weight fraction } \\
\phi[\mathrm{wt} \%]\end{array}$ & $\begin{array}{c}\mathrm{M}_{\mathrm{e}} \\
{[\mathrm{kg} / \mathrm{mol}]}\end{array}$ & $\mathrm{Z}$ & $T_{\mathrm{g}}\left[{ }^{\circ} \mathrm{C}\right]$ \\
\hline \multirow{4}{*}{ PS820 - OS9 } & 50 & 30 & 27.3 & 102.3 \\
\cline { 2 - 5 } & 40 & 37.5 & 21.9 & 101.3 \\
\cline { 2 - 5 } & 30 & 50 & 16.4 & 100.7 \\
\cline { 2 - 5 } & 20 & 75 & 10.9 & 99.8 \\
\cline { 2 - 5 } & 10 & 300 & 2.7 & 98.6 \\
\hline PS389 - OS9 & 5 & 37.5 & 10.4 & 101.7 \\
\cline { 2 - 5 } & 20 & 37.5 & 7.0 & 101.2 \\
\hline PS261 - OS9 & 40 & 75 & 3.5 & 99.8 \\
\hline PS820 - PS256 & 10 & 15 (melt) & & - \\
\hline PS820 - PS256 & 30 & 15 (melt) & & - \\
\hline PS820 - PS389 & 10 & & & \\
\hline
\end{tabular}

As shown in Figure 1, the glass transition temperatures of the polymeric systems depend linearly on the weight fraction of the long chains, $\phi$. This is in agreement with the Fox equation $^{34}$, which describes the glass transition temperature of miscible blends,

$$
\frac{1}{T_{\mathrm{g}}}=\frac{\phi}{T_{\mathrm{g}, P S}}+\frac{1-\phi}{T_{\mathrm{g}, O S}}
$$

Here $T_{\mathrm{g}, P S}=106.6^{\circ} \mathrm{C}$ is the glass transition temperature of the high molar mass $\mathrm{PS}$, and $T_{\mathrm{g}, O S}=$ $97.8^{\circ} \mathrm{C}$, is that of the OS9. For the latter, the experimental glass temperature shown in Table 1 has not been used because it is abnormally low compared with the other data in the set. This discrepancy can be explained by the presence of residual solvent coming from an improper drying of the sample. Instead, this value was extrapolated from the linear fitting of all the other experimental results. In order to compute a precise value of $T_{\mathrm{g}, O S}$, experimentally measured glass transition temperatures of solutions from ref [33] have been added to the dataset. 


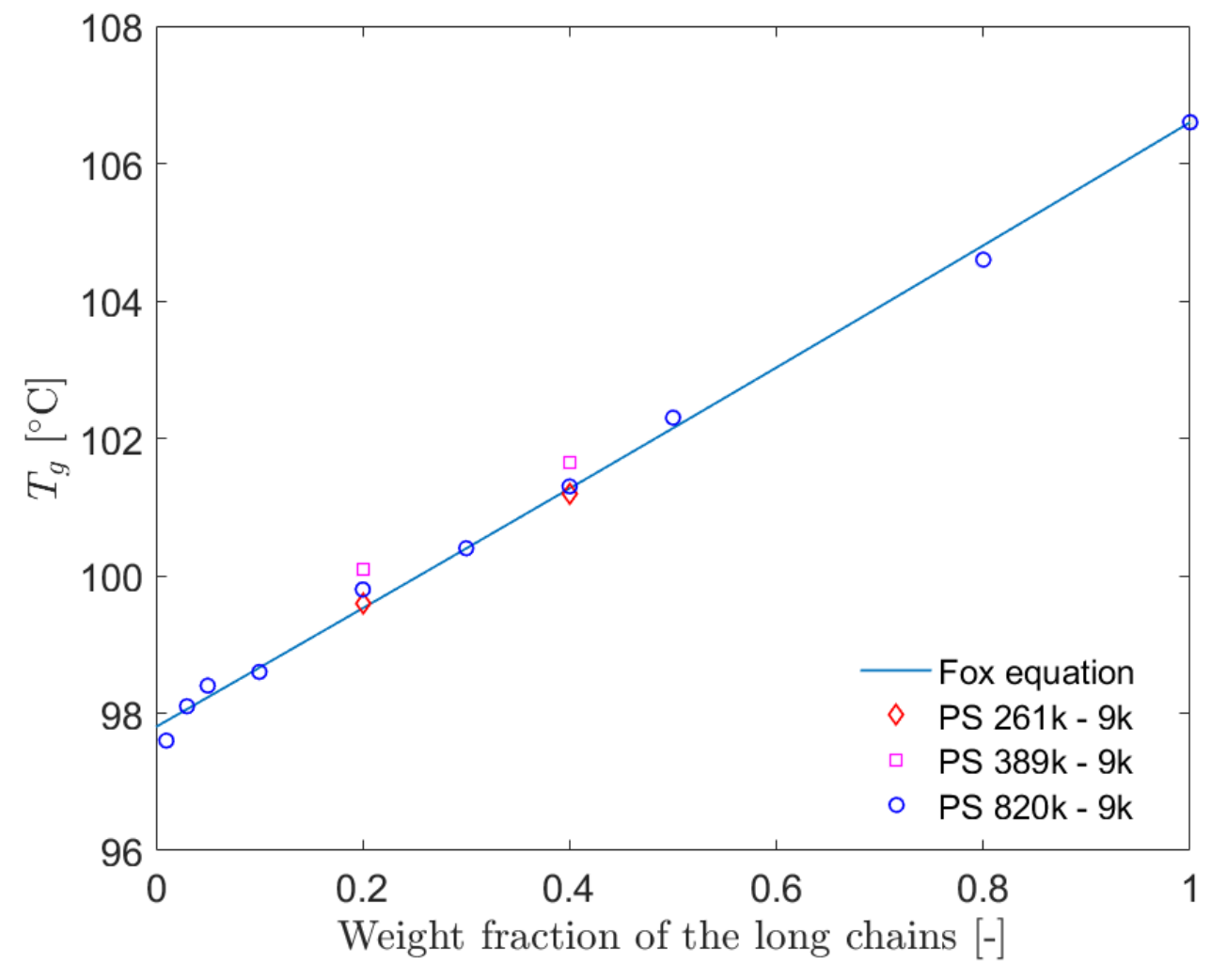

Figure 1 : Glass transition temperatures of PS systems as a function of the weight fraction of the long chains. The symbols are the experimental results and the solid line is the predictions of the Fox equation ${ }^{34}$. Data for PS $820-$ $9 k$ are taken from ref [33]

\section{II.3. Mechanical spectroscopy}

Rheological properties of the PS systems have been measured using two different rheometers: a MCR 301 (Anton Paar, Austria) rheometer to measure the LVE properties, and a filament stretching rheometer (FSR) (VADER-1000, Rheo Filament, USA) to obtain the transient elongational responses. All the samples were dried at $70{ }^{\circ} \mathrm{C}$ overnight before being placed into disc-shaped, in-house developed molds. In the mold, the samples were molten, pressed and annealed at $160{ }^{\circ} \mathrm{C}$ under vacuum. For the linear measurements, a mold that yields disks of 8 $\mathrm{mm}$ diameter $\mathrm{D}_{0}$ and a thickness $\mathrm{t}_{0}$ of $0.8 \pm 0.2 \mathrm{~mm}$ was used. For the extensional measurements, a $6 \mathrm{~mm}$ mold was used that yielded disks with a final aspect ratio $\Lambda_{0}\left(=2 \mathrm{t}_{0} / \mathrm{D}_{0}\right)$ of $0.5 \pm 0.15 \mathrm{~mm}$. 


\section{Linear viscoelastic properties}

Small amplitude oscillatory shear (SAOS) flow measurements have been performed using a stainless steel $8 \mathrm{~mm}$ parallel plate geometry with a convection oven for temperature control $\left(\sim 0.1{ }^{\circ} \mathrm{C}\right)$ under $\mathrm{N}_{2}$ atmosphere. For each sample, an amplitude sweep was carried out to determine the LVE region. Then, at each selected experimental temperature, a time sweep was done to ensure the sample was stabilized. Once stable conditions were achieved, a frequency sweep was performed at different temperatures ranging from 130 to $170{ }^{\circ} \mathrm{C}$ to determine the LVE data of each sample. Subsequently, for each polymeric system the data were shifted onto a single master curve at a reference temperature $T_{r e f}$ of $130{ }^{\circ} \mathrm{C}$ using the time-temperature superposition (TTS) principle. No vertical shift has been considered as the density change over the range of temperature used is negligible. In order to check the validity of the experimental shift factors of the PS melt, they are compared with those predicted by the Williams-LandelFerry (WLF) equation ${ }^{35}$ :

$$
\log _{10} a_{T}=\frac{-c_{1}^{0}\left(T-T_{r e f}\right)}{c_{2}^{0}+\left(T-T_{r e f}\right)}
$$

with $c_{1}^{0}=8.99$ and $c_{2}^{0}=81.53 \mathrm{~K}$. Data for $c_{1}^{0}$ and $c_{2}^{0}$ is taken from ref [24].

Due to the different glass transition temperatures of the PS solutions (see Table 2), their horizontal shift factors are different and have a smaller temperature dependence than those of their parent melts. Indeed, as $T_{\text {ref }}-T_{\mathrm{g}}$ increases, the activation energy decreases and so does the dependence of the relaxation times with the temperature. Despite this, as demonstrated by Wagner ${ }^{12}$ and Liu et al. ${ }^{36}$, it is still possible to draw a mastercurve for the shift factors of both the melts and the solutions. This can be done by multiplying the shift factors of the solutions by a correction factor $a_{T_{g}}$ which takes into account the effect of the different $T_{\mathrm{g}}$. This shift $a_{T_{g}}$ can be computed by a WLF-like equation: 


$$
\log _{10} a_{T_{g}}=\frac{-c_{1}^{0}\left(T_{g, \text { melt }}-T_{g}\right)}{c_{2}^{0}+\left(T_{g, \text { melt }}-T_{g}\right)}
$$

with the glass transition temperature $T_{g}$ of the solutions and the glass transition temperature of their corresponding parent melt $T_{g, \text { melt }}=106.6^{\circ} \mathrm{C}$.

As shown in Figure 2, when expressed as a function of $T-T_{g}$, all the corrected shift factors superpose well with the WLF equation.

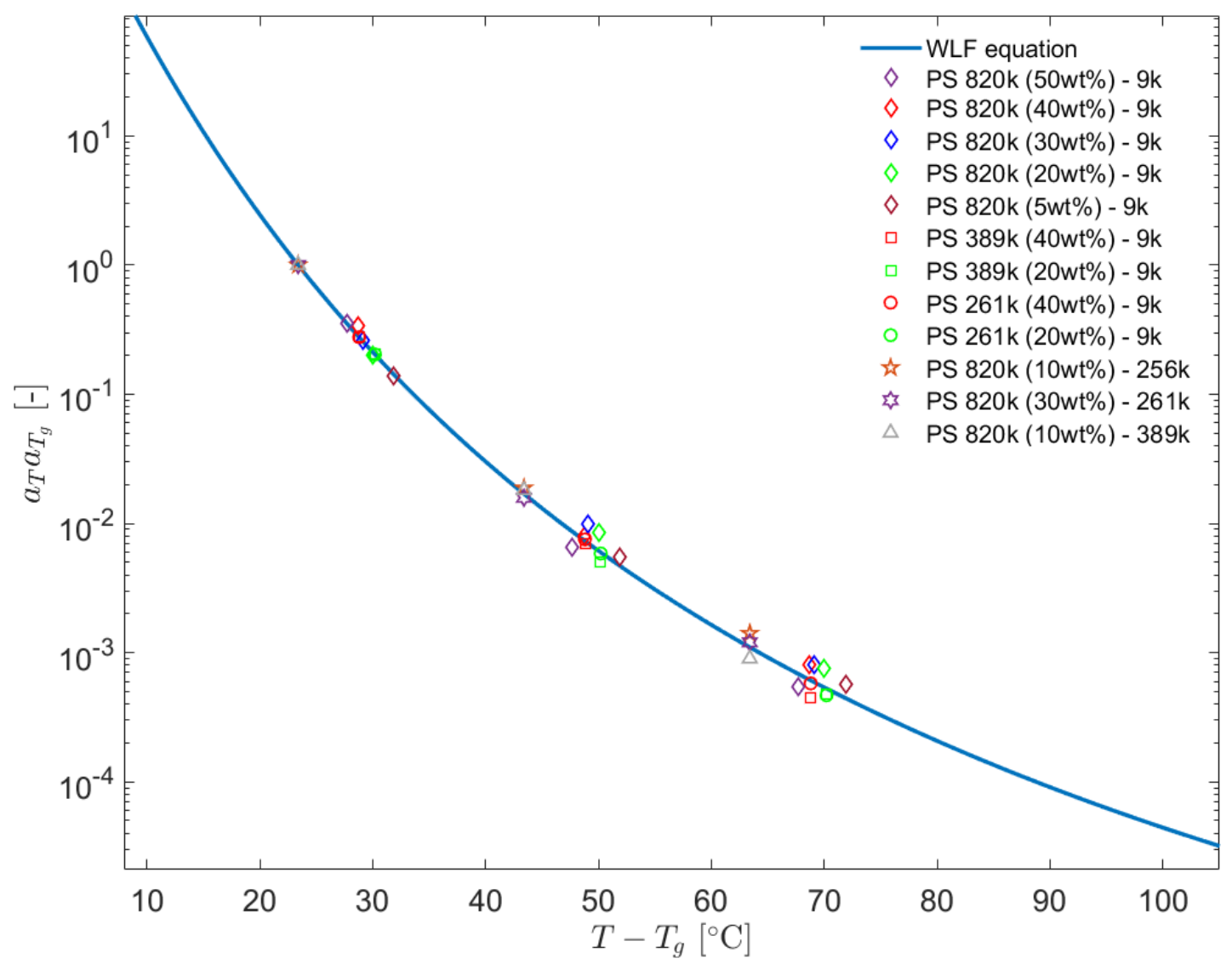

Figure 2: Corrected shift factors $a_{T} a_{T_{g}}$ of PS systems as a function of $T-T_{g}$ at the reference temperature $T_{\text {ref }}=130{ }^{\circ} \mathrm{C}$. The symbols are the experimental results and the solid line is the predictions of the WLF equation. Data for PS 820 - 9k systems are taken from ref [33] 
This allows to validate the experimental shift factor $a_{T}$ and the values of $c_{1}^{0}$ and $c_{2}^{0}$ at $T_{r e f}$. With this, one can frequency-shift the LVE responses of the different polymeric systems onto a temperature $T_{0}=T_{g}+23.4{ }^{\circ} \mathrm{C}$ to compare them at iso- $T_{g}$ conditions.

\section{Viscoelastic response under uniaxial elongation}

Extensional stress measurements have been performed on the FSR using stainless steel $6 \mathrm{~mm}$ plates with an oven for temperature control $\left(\sim 0.1^{\circ} \mathrm{C}\right)$ under constant nitrogen gas supply. All filament stretching measurements were carried out using the subsequent protocol, inspired by $\operatorname{ref}[7]$.

Once the temperature in the oven is raised to $165^{\circ} \mathrm{C}$, the sample is placed inside the rheometer on the bottom plate. After melting the polymer, the sample is slightly compressed by lowering the top plate to ensure a proper adhesion between the sample and the geometries in order to avoid detachment of the sample from the geometry during stretching. This squeezing was continuously interrupted to give the sample time to relax. Afterwards, the sample is prestretched to a desired smaller radius, in order to decrease the force build-up during the actual stretching and thus to avoid detachment during the main stress measurement. Again, similarly to the compression, the pre-stretch several times temporarily halted to allow the sample to relax. This is performed until the starting radius $R_{0}$ is reached, which ranges from 1.2 to $3.5 \mathrm{~mm}$. The choice of the radius $R_{0}$ is based on the type of the sample and the experimental conditions. When high strain rates are applied to highly viscous samples, the force build-up is so high that rupture at both ends of the filament cannot be prevented without reducing the radius $R_{0}$. On the other hand, when a low-viscous sample is stretched at low strain rates, a sufficiently high diameter is needed to avoid a too noisy signal. Finally, the temperature of the oven is lowered to the temperature of the experiment. Once the sample is relaxed, it is stretched at a constant 
strain rate between $3 \times 10^{-5}$ and $0.2 \mathrm{~s}^{-1}$. The strain $\epsilon$ in the following is defined as the Hencky strain

$$
\epsilon_{H}(t)=-2 \log \left(\frac{R(t)}{R_{0}}\right)
$$

The diameter $2 R(t)$ at the mid filament plane was monitored by a laser micrometer to constantly adjust via a control scheme ${ }^{37}$ the vertical speed of the top plate to keep the strain rate constant. During these measurements, the force $F(t)$ exerted by the polymeric filament on the load cell was recorded. During the start-up of elongational flow, part of the force comes from the radial variation due to the shear component of the deformation field, which can be compensated by a correction factor, as mentioned by H. K Rasmussen et al. ${ }^{38}$ Subsequently for larger strains, the correction vanishes, and the radial variation of the stress in the symmetry plane becomes negligible. ${ }^{39}$ Assuming the mid filament has planar as well as axial symmetry, the extensional stress growth coefficient $\eta_{E}^{+}(t)$ can be calculated via

$$
\eta_{E}^{+}(t)=\frac{F(t)-\frac{m g}{2}}{\pi R(t)^{2} \dot{\varepsilon}} A\left(\Lambda_{0}\right)
$$

where $m$ is the mass of the sample, $g$ is the gravitational acceleration and $A\left(\Lambda_{0}\right)$ is the correction factor of the shearing contribution. This latter ensures less than $3 \%$ deviation from the correct initial stress if $\Lambda_{0}>0.3 .{ }^{37}$ The resulting extensional curves have been averaged to decrease the importance of noise and to make the curves smoother. In addition to the stress growth coefficient presented in the next sections, the measured extensional stresses as a function of the Hencky strain of all the samples are available as Supplemental Material.

\section{Results and Discussion}

\section{III.1. Linear rheology}


Figure 3 and 4 shows the storage and the loss moduli of the PS systems at iso- $T_{g}$ condition. The LVE properties of the melts and PS820 - OS9 can be found in ref [33]. In order to model the LVE responses of the long chains diluted in the oligomers with well separated relaxation times, the modified TMA tube-based model developed by van Ruymbeke et al. ${ }^{40}$ was used. A good prediction of the experimental data is obtained at $T=T_{g}+23.4^{\circ} \mathrm{C}$, by considering the molar mass between two entanglements, $M_{e}$, to be equal to $15 \mathrm{~kg} / \mathrm{mol}$, the Rouse time of an entanglement segment to be $\tau_{e}=0.55 \mathrm{~s}$, and the plateau modulus as $G_{N}^{0}=230 \mathrm{kPa}$, consistent with the work of Shahid et al. ${ }^{33}$ The molecular weight of a Kuhn segment is equal to $M_{0}=$ $833 \mathrm{~g} / \mathrm{mol}^{41}$ and the dynamic dilution exponent $\alpha$ has been fixed to $1 .{ }^{42}$ These values allow to determine consistently the different characteristic times of the flow dynamics of the polymer on a physical basis. The Rouse times $\tau_{R}=Z^{2} \tau_{e}$ of the different chains, which characterizes the so-called stretch relaxation time, are shown in Table 3. They have been computed at two different iso- $T_{g}$ condition as in this work the $\Delta T_{g}$ is set to $31.4{ }^{\circ} \mathrm{C}$ in section III.2, and $23.4{ }^{\circ} \mathrm{C}$ in the other sections. The number of entanglement segments per chain, $Z$, was calculated as the ratio $M_{w} / M_{e}$. The reciprocal Rouse time $\tau_{R}^{-1}$, which is approximately half of the onset of stretching in the standard tube model ${ }^{3}$, has also been included in the Table. Nevertheless, it should be considered with care, as the criteria for the critical strain rate for chain stretching are still under debate. ${ }^{43,44}$

Table 3. Rouse times and reciprocal Rouse times of the chains

\begin{tabular}{|l|c|c|c|c|}
\hline & \multicolumn{2}{|c|}{$\Delta T_{g}=23.4^{\circ} \mathrm{C}$} & \multicolumn{2}{c|}{$\Delta T_{g}=31.4^{\circ} \mathrm{C}$} \\
\hline & $\tau_{R}[\mathrm{~s}]$ & $\tau_{R}^{-1}\left[\mathrm{~s}^{-1}\right]$ & $\tau_{R}[\mathrm{~s}]$ & $\tau_{R}^{-1}\left[\mathrm{~s}^{-1}\right]$ \\
\hline PS820 & 1643.6 & $6 \times 10^{-4}$ & 258.5 & 0.0039 \\
\hline PS389 & 369.9 & $2.7 \times 10^{-3}$ & 58.2 & 0.017 \\
\hline PS261 & 166.5 & $6 \times 10^{-3}$ & 26.2 & 0.038 \\
\hline OS9 & 0.2 & 5 & 0.03 & 33.34 \\
\hline
\end{tabular}


As illustrated in Figure 3 and 4, all PS systems overlap at high frequencies, thus confirming that the iso- $T_{g}$ condition is fulfilled. Indeed, in the high frequency portion of the moduli the polymer chains relax only by a Rouse process, which starts locally and is not influenced by the length of the chain or the dilution. This regime continues until either the whole chain is relaxed at a frequency $\omega_{R} \sim \tau_{R}^{-1}$ for the OS or it is interrupted by the entanglements at a frequency $\omega_{e} \sim \frac{\phi^{2}}{\tau_{e}}$ for the long chains. For the solutions diluted at $20 \mathrm{wt} \%$ the difference in the high frequency response of the OS and long chain polymer is clearly perceptible: While the high frequency regime is dominated by the OS relaxation, the frequency window between 1 and 20 $\mathrm{rad} / \mathrm{s}$ corresponds to a transition state between the relaxation of the OS of molar mass $9 \mathrm{~kg} / \mathrm{mol}$ and the Rouse relaxation of the long chains, up to the relaxation of segments between two entanglements of mass $\frac{M_{e}}{\phi}=60 \mathrm{~kg} / \mathrm{mol}$, taking place near $\omega_{e} \approx 0.73 \mathrm{rad} / \mathrm{s}$.

At intermediate frequencies, the solutions display a rubbery plateau, which gradually decreases with the level of chain dilution. It should be noted that, for the most diluted solutions, the plateaus are barely noticeable due to the very small number of entanglements. (i.e. $Z \sim 3-4$ for PS261k (20 wt \%) and 5 for PS389k (20wt\%)).

Finally, the terminal relaxation time decreases with decreasing concentration as predicted by the dynamic tube dilution theory ${ }^{45}$.

These experimental data are then used to create LVE envelopes that reconstruct the stress growth coefficient of elongational measurements performed at very low strain rates. Any upward deviation of the transient extensional data from these envelopes indicates strain hardening. 


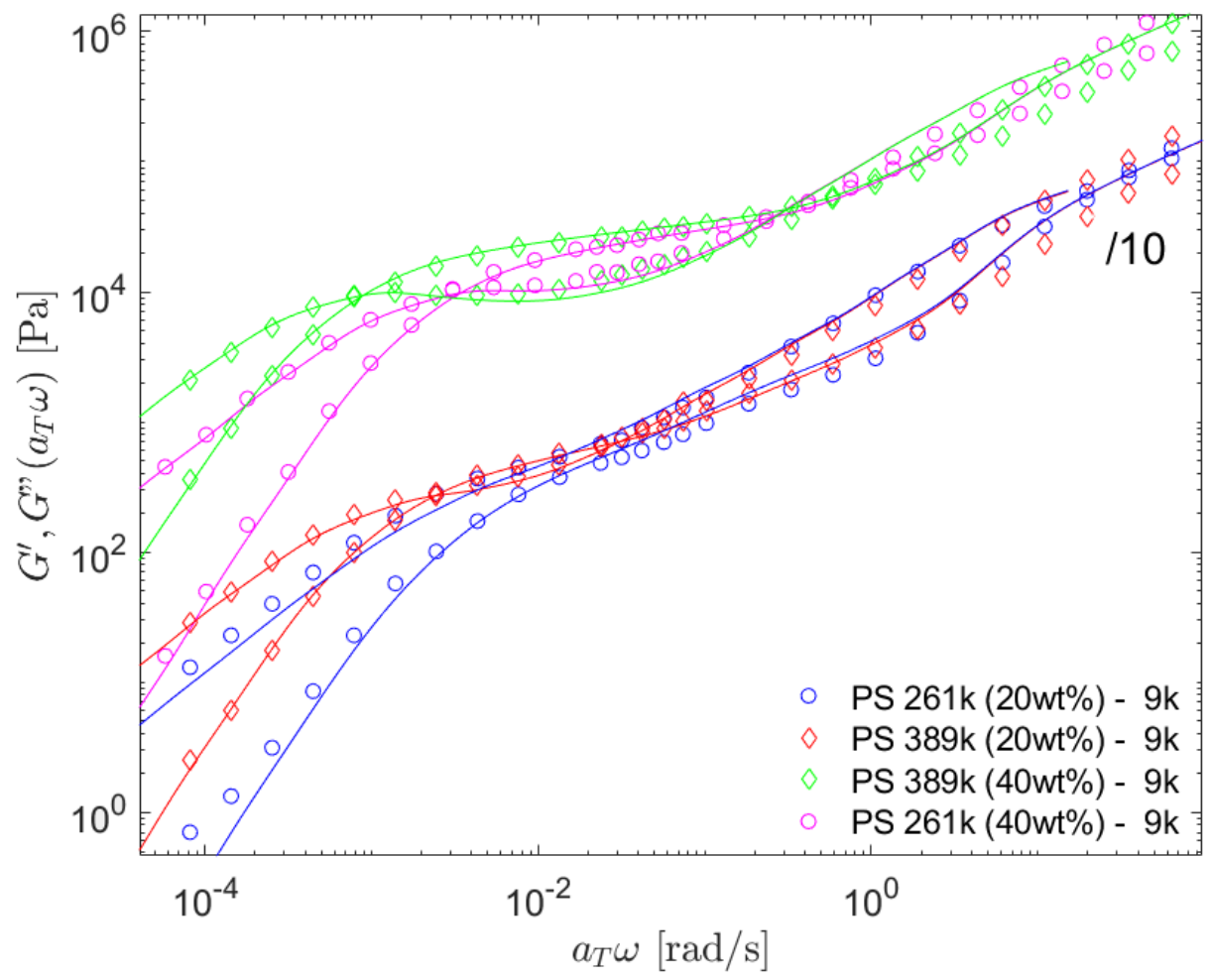

Figure 3: Linear viscoelastic response of the PS $261 k-9 k$ and $P S 389 k-9 k$ solutions at $T=T_{g}+23.4{ }^{\circ} \mathrm{C}$. For clarity, the data of the PS $261 k(20 w t \%)-9 k$ and PS 389k (20wt\%) - 9k systems have been downshifted by 1 decade. The symbols are the experimental results and the solid lines are the predictions of the TMA tube-based model $4^{40}$.

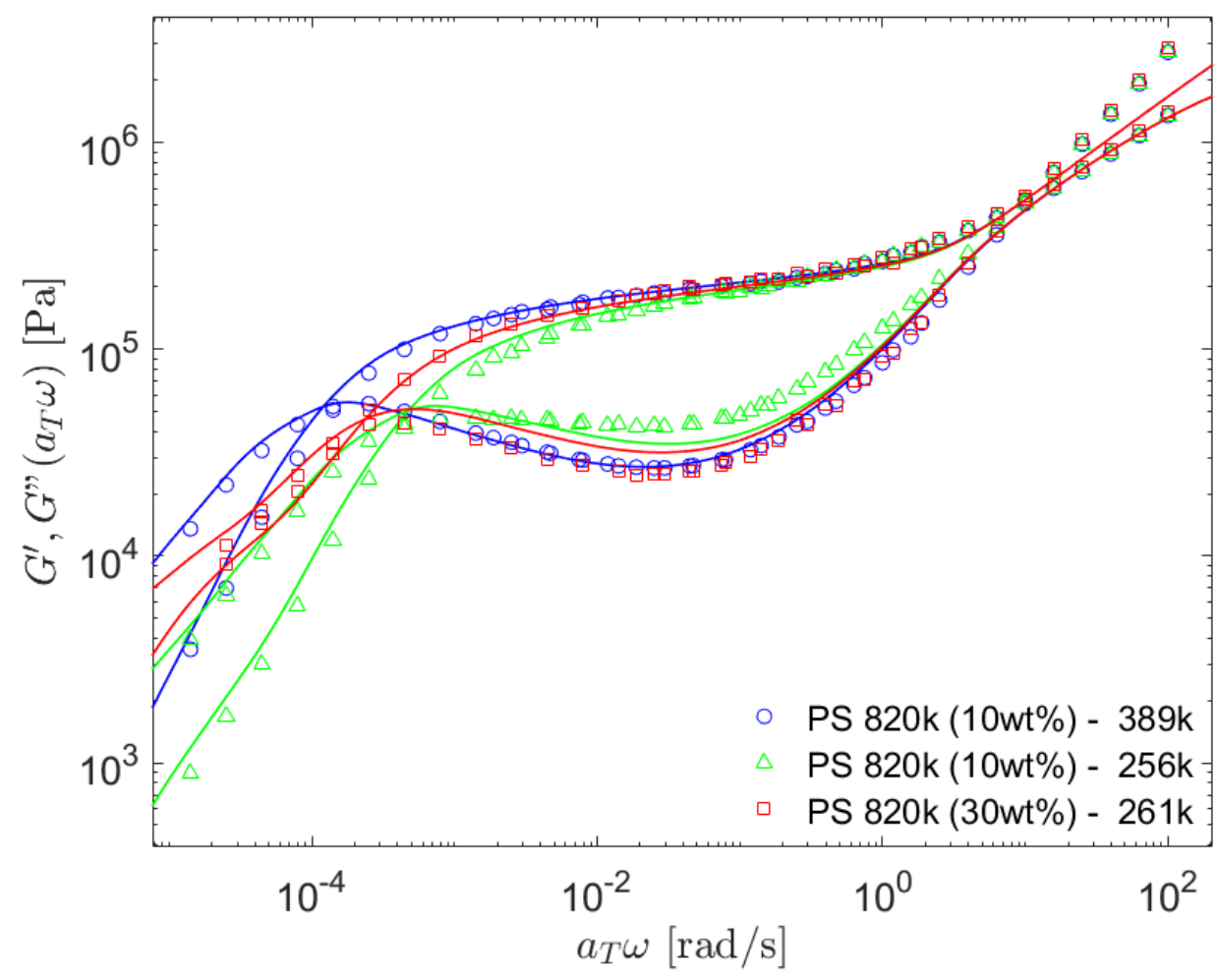


Figure 4: Linear viscoelastic response of the PS $820 k(10 w t \%)-389 k$, PS $820 k(10 w t \%)-256 k$ and PS $820 k$ $(30 w t \%)-261 k$ systems at $T=T_{g}+23.4{ }^{\circ} \mathrm{C}$. The symbols are the experimental results and the solid lines are the predictions of the TMA tube-based model ${ }^{40}$.

\section{III.2. Elongation viscosity: From the melt state to solution}

Figure 5 shows the experimental results of the transient elongational measurements for the PS solutions with varying concentration, as well as the corresponding LVE envelopes. At very low strain rates (typically at strain rates smaller than the reciprocal reptation time), the transient elongational viscosity follows the LVE envelopes. This is exemplarily demonstrated for two cases of elongational flow measurements at very low rates, shown in Figure 5 a).

At high strain rates, an experimental deviation from the LVE responses can be observed at very short times. There are mainly two reasons to that. When starting the experiments, there is still a small residual stress due to the required pre-stretching of the polymer that cannot relax within reasonable experimental timescales. In addition, the fluctuations inherent in the control loop implemented in the FSR causes the force to exhibit oscillations at short times. At the start-up of the experiment, these oscillations are significant as the flow is not stabilized due to the limitations in the control of the FSR for starting the plate displacement from the rest state. However, this deviation only influences the data at short times, the steady states and data at longer times being unaffected as the stress involved is few orders of magnitude higher. Apart from that, the transient viscosity of the solutions first follows the LVE responses, and then precipitously deviates before reaching a steady state. For all the strain rates presented here, at least the onset of the steady state value was reached as shown in Figure S1 and S2 of the Supplemental Material (SM). 

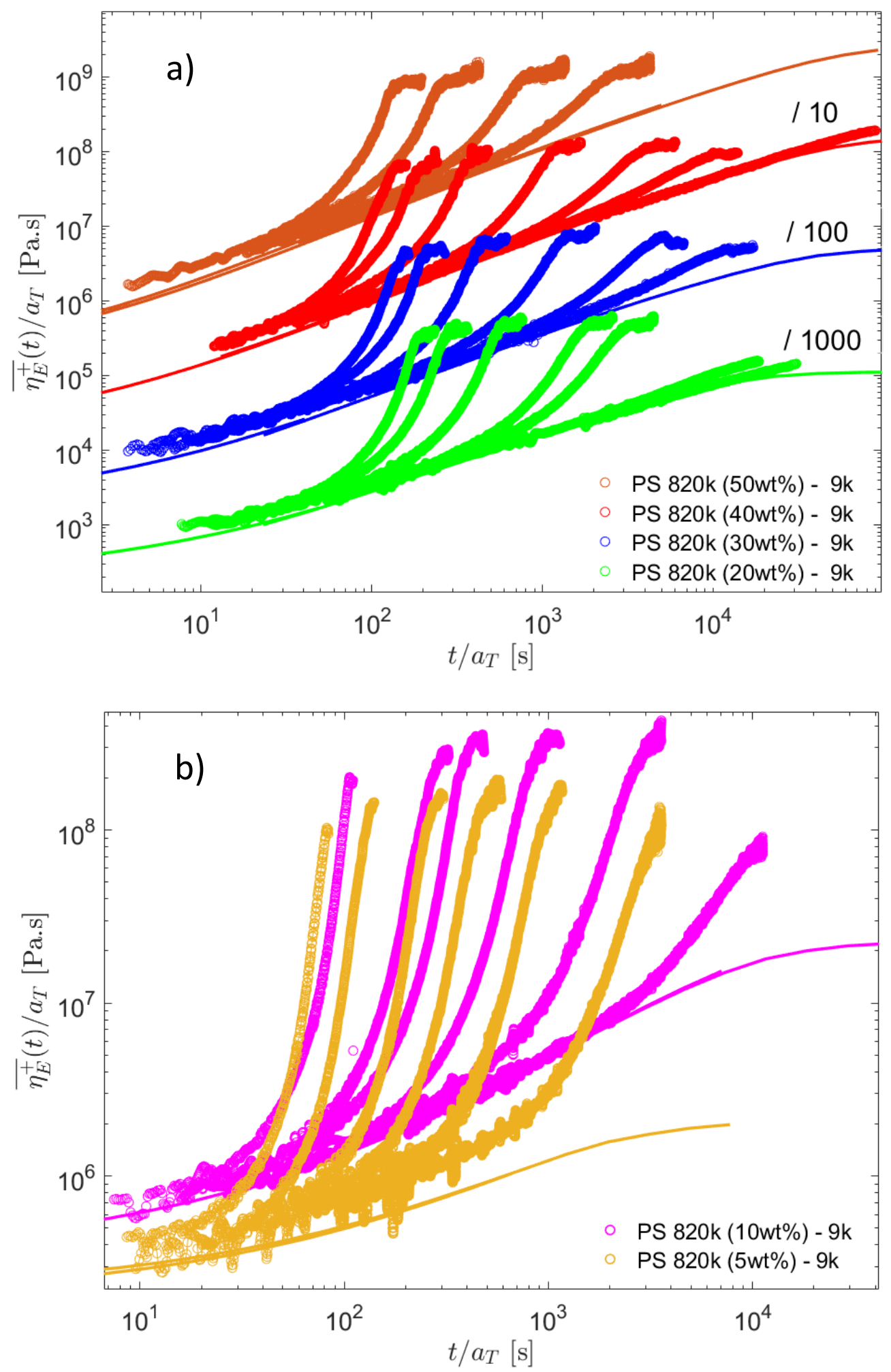

Figure 5: a) Stress growth coefficient for solutions. Data were measured for different strain rates at $T=130^{\circ} \mathrm{C}$. For Figure 5a), the strain rates are from right to left 0.001, 0.003, 0.01,0.03, 0.06, $0.09 \mathrm{~s}^{-1}$, except for PS $820 \mathrm{k}$ (50 wt\%) for which the strain rates are from right to left 0.003, 0.01, 0.03, $0.06 \mathrm{~s}^{-1}$. Additionally, for PS $820 \mathrm{k}(40$ $w t \%)$ and PS 820k (20wt\%), LVE envelopes have also been measured in elongational flow at 0.0001 and 0.00003 $s^{-1}$ respectively. For Figure 5b), the strain rates are from right to left 0.003, 0.01, 0.03, 0.06, 0.09, $0.2 s^{-1}$ for 
PS 820k (10 wt\%) and 0.01, 0.03, 0.06, 0.1, 0.2, $0.3 \mathrm{~s}^{-1}$ for PS 820k (5 wt\%). All the data have been subsequently shifted to $T=T_{g}+23.4^{\circ} \mathrm{C}$. For clarity, some of the extensional data has been downshifted by 1, 2 or 3 decades as indicated.

From these figures, it can be observed that the strain hardening depends on the level of the dilution, and that the ratio between steady state viscosity in the nonlinear regime and the zero elongation viscosity, $\frac{\eta_{E, s t}}{3 \eta_{0}}$, increases significantly with decreasing concentration levels. The effect is more obvious in Figure 6, where the steady state viscosity values of Figure 5 are plotted as a function of the strain rate. It is observed that sample PS820k (50 wt\%) shows the least strain hardening behavior. Furthermore, the corresponding steady state viscosities remain below the zero-elongation viscosity $3 \eta_{0}$ for all measured strain rates, thus showing transient thickening but steady thinning behavior. On the other hand, the most diluted sample PS820k (5 $\mathrm{wt} \%$ ) shows the most prominent strain hardening behavior and, with more than two orders of magnitude, the largest ratio between the steady state elongational viscosity and $3 \eta_{0}$, thus exhibiting transient thickening and steady thickening followed by a steady thinning behavior. 


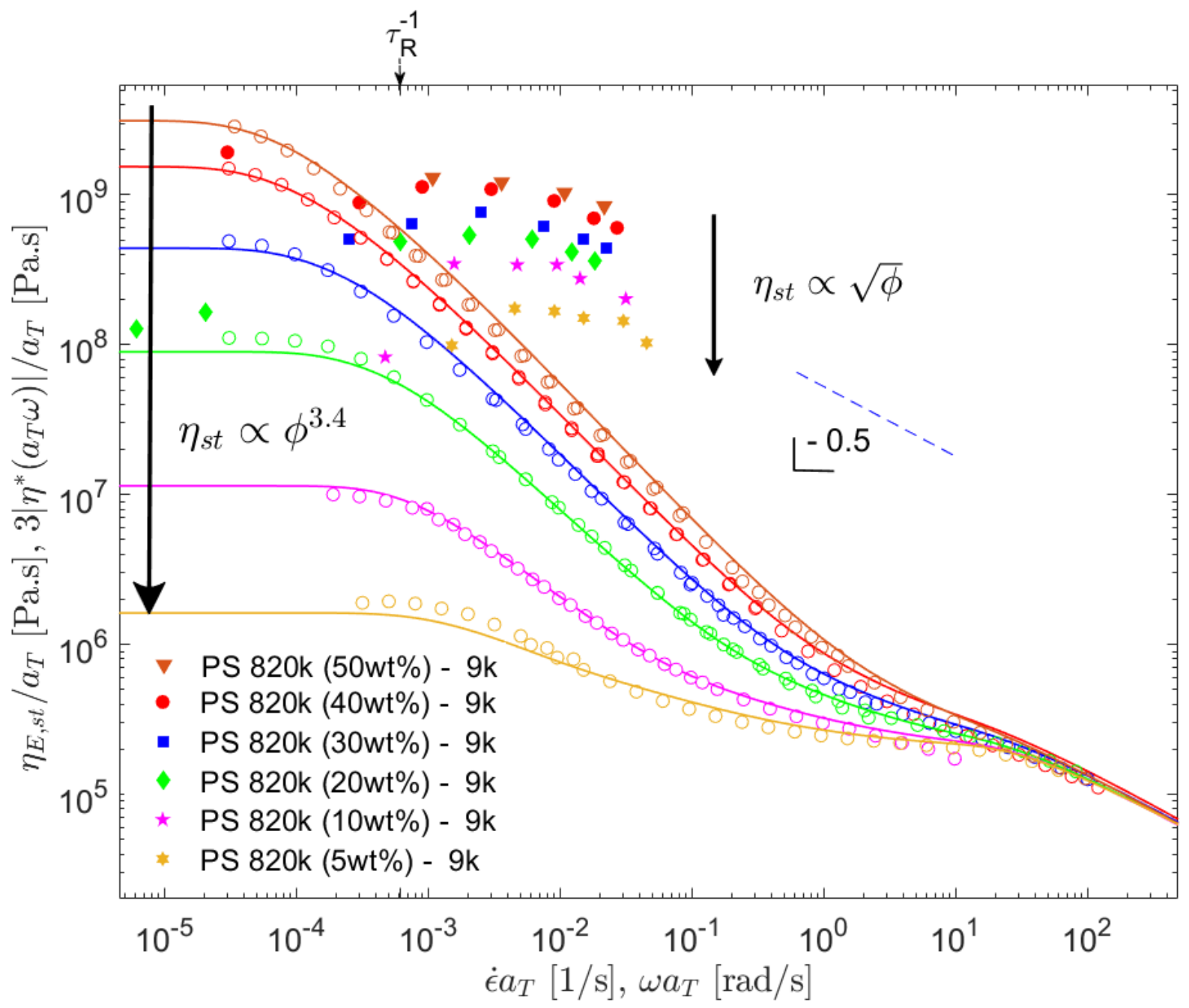

Figure 6: Elongational steady state viscosity (closed symbols) of PS solutions and PS melt as a function of the strain rates. The corresponding LVE data (open symbols) are also shown. The solid lines represent the predictions of the TMA tube-based model. ${ }^{40}$ Data are respectively measured or shifted to $T=T_{g}+23.4{ }^{\circ} \mathrm{C}$.

At high strain rates, all steady state viscosity values show a similar decrease with elongation rate with a scaling of $\eta_{E, s t} \propto \dot{\varepsilon}^{-0.5}$. In the literature, a similar scaling has already been encountered for different chain architectures (i.e. linear ${ }^{5}$ and $\operatorname{star}^{46}$ PS chains and PS rings ${ }^{47}$ ), for entangled ${ }^{5}$ and diluted systems ${ }^{48}$, and for polymer of different natures (i.e. $\mathrm{PS}^{5}$, Polyisoprene $^{48}$ (PI), Low Density Polyethylene ${ }^{49}$ (LDPE) and Poly(methyl methacrylate) ${ }^{50}$ (PMMA)). However, the last point on polymer nature is in minor variance with the recent work 
of Morelly et $a l .{ }^{51}$, who found slightly different power-law exponents for PMMA, PS and poly(tert-butylstyrene) (PtBS).

An inspection of Figure 6 reveals three distinct regimes with different dependence of the steady state elongational viscosity on the concentration.

\section{A. Low strain rates:}

At low strain rates the PS solutions follow the LVE envelopes, the steady state viscosity $\eta_{E, s t}$ is therefore well predicted by the Doi-Edwards theory ${ }^{1}$. If we assume that the contribution of the short chains is negligible, $\eta_{E, s t}$ has the following scaling:

$$
\eta_{E, s t}=3 \eta_{0} \propto \frac{\rho N_{A}}{M_{0}} \zeta_{0}\left(\frac{N^{x}}{N_{e}^{x-1}}\right) \propto M_{w}^{x} \phi^{x}, \quad x \in[3,3.4]
$$

where $\rho$ is the mass density, $N_{A}$ is the Avogadro number, $M_{0}$ is the mass of a Kuhn segment, $\zeta_{0}$ is the friction coefficient of a Kuhn segment, $N$ is the number of Kuhn segments of a long chain, and $N_{e}=\frac{M_{e, 0}}{M_{0} \phi}$ is the number of Kuhn segments between two entanglements, with $M_{e, 0}$ being the molar mass between two entanglements in the melt state. Thus, in the linear regime, the steady viscosity scales with the number of entanglement segments, $Z=\frac{\phi M_{w}}{M_{e, 0}}$. The $\phi$ dependence of the steady viscosity can be found by considering that $\eta_{0}$ is well approximated by $G_{N}^{0} \tau_{d}$. Indeed, the plateau modulus $G_{N}^{0}$ scales with $\phi^{2}$ (if we consider that the dilation exponent is equal to 1), while the terminal relaxation time $\tau_{d}$ scales with $\phi^{1-1.4}$, since the reptation time of the chains scales with $\phi^{1}$ and their fluctuations times scales with $\phi^{2}$. As for polymer melts, different scalings are thus obtained depending on the importance of the contour length fluctuations, these last ones depending on the entanglement state of the chains. ${ }^{33}$

\section{B. High strain rates:}


At high strain rates the polymer chains are predominantly stretched and exhibit a strongly nonlinear behavior. In this regime, the concentration dependence is much lower than in the linear regime. In order to assess the influence of the concentration on the steady state extensional viscosity, linear regressions were performed on the data of the polymeric systems. The data set includes all the highest strain rates measured for all the PS solutions measured in this work. By assuming that the strain rates are sufficiently high, so that they are in the high stretch regime, and that the steady state viscosity evolves linearly with the molar mass (see Section III.3), the steady state extension viscosity $\eta_{E, s t}$ can be expressed as

$$
\eta_{E, s t}=A G_{N, m e l t}^{0} \sqrt{\frac{\tau_{R}}{\dot{\epsilon}}} \phi^{x}
$$

where $A$ and $x$ are the parameters of the regression. In this expression, the Rouse time is chosen to normalize the elongation rate, as it scales with the square of the molar mass of the chains. In order to increase the accuracy of the regression, the steady state viscosities of two polymer melts from ref [7] have been added to the dataset.

Figure 7 shows that a good agreement could be found by using Equation 7, with an $\overline{R^{2}}$ of 0.933 . The values of the exponent $x$ of the equation with $95 \%$ coefficient bounds are $x=0.5 \pm 0.1$.

Thus, from this result, we conclude that, at high strain rates, the steady state viscosity of polymeric solutions rather evolves with the square root of the concentration. It must be noted here that this 0.5 scaling must be taken as indicative in view of the scatter in the data. However, the concentration scaling of the steady viscosity is certainly closer to a $\sqrt{\phi}$ dependence than to a $\phi$ dependence. 


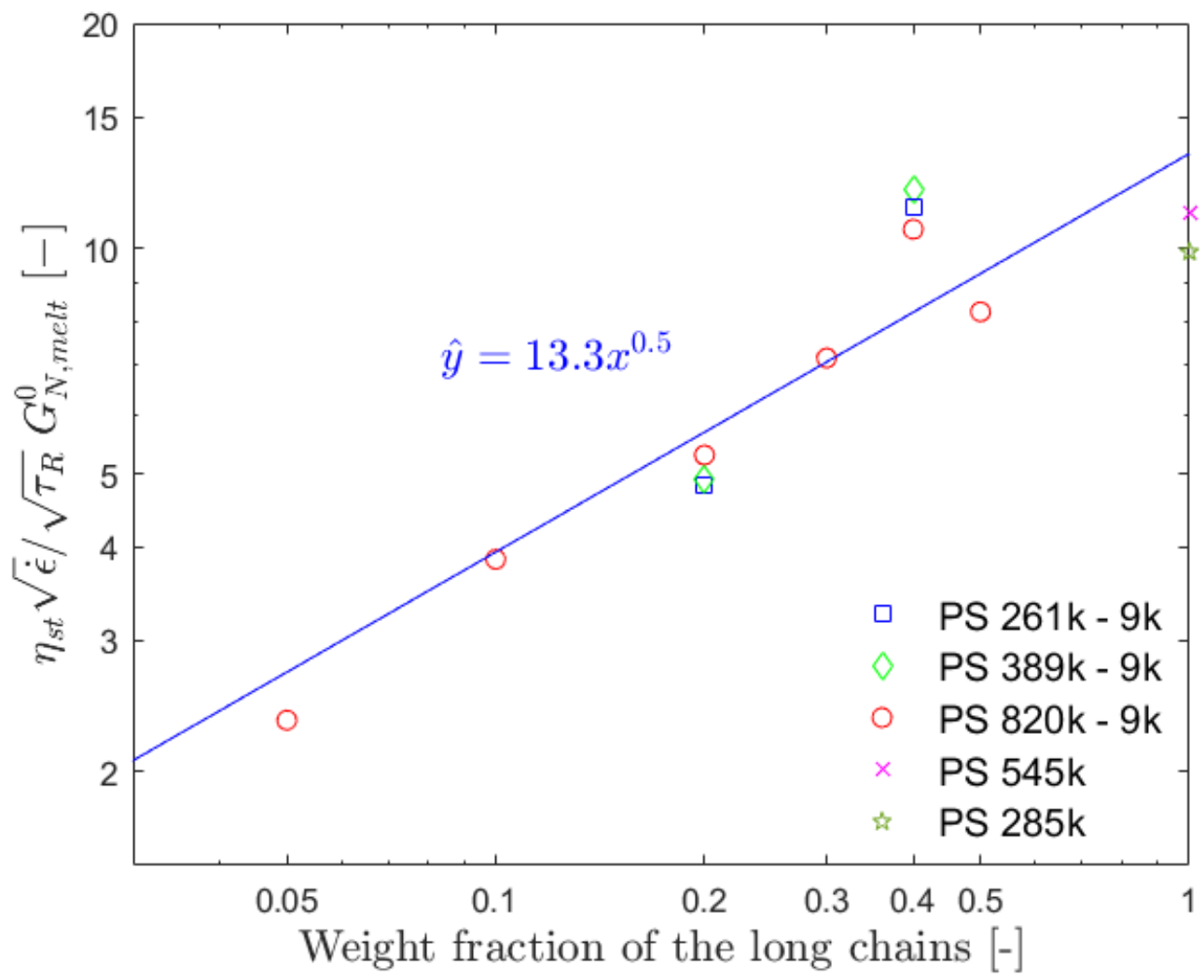

Figure 7: Assessment of the influence of the concentration on the steady state elongation viscosity.

It must be noted that this experimentally observed scaling was not expected from a modelling point of view, and cannot be explained by the classical DEMG theory ${ }^{3}$. In this theory, the steady state viscosity for fully oriented chains is given by the following equation:

$$
\eta_{E, s t}=3 G_{N}^{0} k(\lambda) \lambda^{2} \dot{\varepsilon}^{-1}
$$

In this equation, the stretch $\lambda$ is defined as the ratio of the length of the primitive path $L_{p p}$ relative to the equilibrium length $L_{e q, 0}=Z \sqrt{N_{e} b^{2}}$. Its maximum value $\lambda_{\max }$ is therefore determined by $\sqrt{N_{e}}$. The coefficient $k(\lambda)$, which is given by the inverse Langevin function, describes the deviation of the FENE spring from the pure Hookean spring. At high strain rates $\left(W i_{R} \gg 1\right)$, the DEMG theory predicts that the steady state viscosity saturates as the chains become nearly fully stretched. The corresponding value of the elongation viscosity in this regime, $\eta_{F E N E}$, is given by $\mathrm{y}^{31,32,51}$ 


$$
\eta_{\text {FENE }}=\frac{1}{12} \frac{\rho N_{A}}{M_{0}} \zeta_{0} N^{2} b^{2}
$$

where $b$ is the length of a Kuhn segment. As a consequence, $\eta_{F E N E}$ is predicted to evolve linearly with $\phi$ as it scales with the long chains density $\rho$. Since this scaling differs from the scaling found experimentally, it is interesting to determine if the samples reach their finite extensibility. Figure 8 shows the ratio of $\eta_{F E N E}$ to the maximum viscosity $\eta_{E \text {, max }}$ obtained for our solutions after $W i_{R}>1$. While by increasing the dilution $\eta_{E, s t}$ draws nearer to $\eta_{F E N E}$, it clearly stays below this limit, indicating that these systems are not fully stretched. However, even if the chain stretch in the steady regime is lower than the finite extensibility, assuming that the chain stretch depends on its Rouse Weissenberg number, $W i_{R}=\dot{\epsilon} \tau_{R}$, one would have expected a $\phi$ - dependence of the steady viscosity in this high strain rate regime. Indeed, the chains have the same Rouse time and are therefore expected to stretch in the same way, whatever their concentration. Only the density should be affected by the dilution of the polymer chains. This $\phi$-dependence can also be found if we consider that the steady viscosity is well approximated by $\eta_{E, s t}=3 G_{N}^{0} \lambda_{m e l t}^{2} \epsilon^{-1}$ in the large elongation rate regime $\left(\dot{\epsilon}>\frac{1}{\tau_{R}}\right)$, since by diluting the sample the plateau modulus is rescaled as $G_{N}^{0} \phi^{2}$, while the stretch factor is rescaled as $\lambda=\frac{L_{p p}}{L_{e q, 0}}=\lambda_{\text {melt }} \frac{L_{e q, 0, m e l t}}{L_{e q, 0}}=\lambda_{\text {melt }} \phi^{\frac{1}{2}}$. 


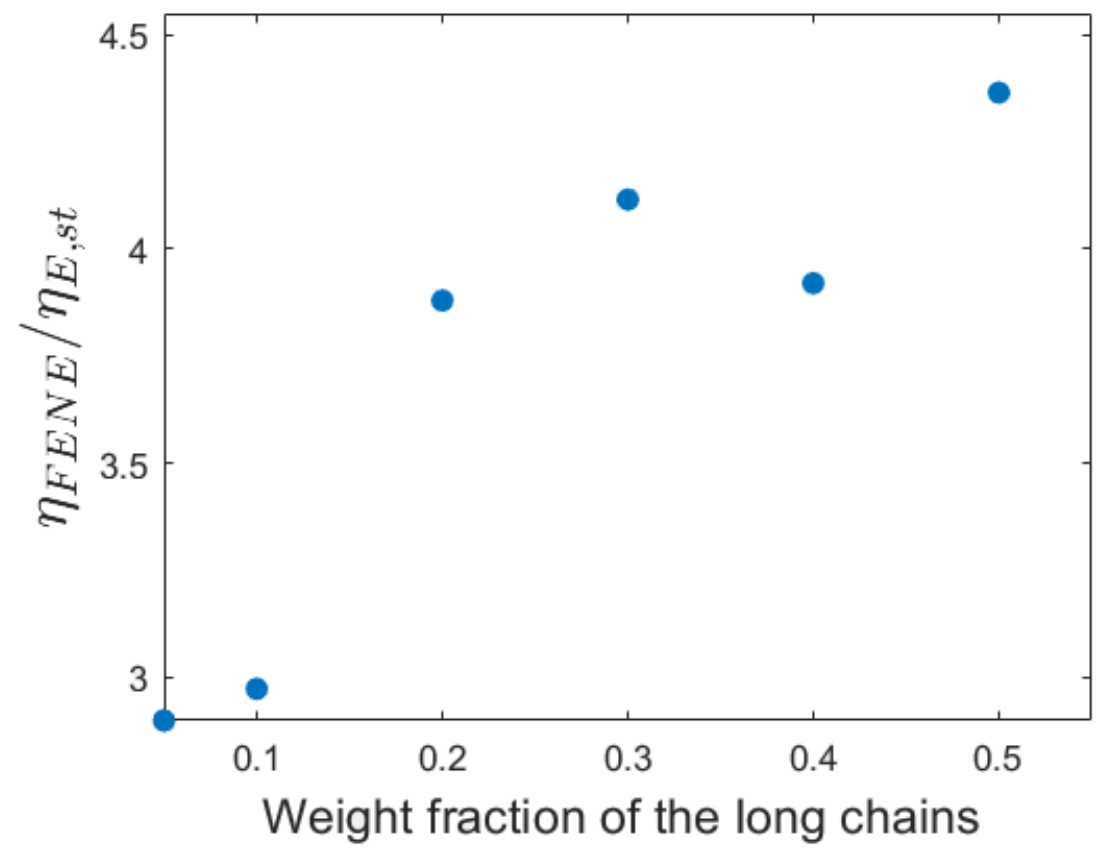

Figure 8: Ratio of $\eta_{F E N E}$ to the maximum steady state viscosity $\eta_{E, \text { st }}$ obtained for the PS820k - 9k solutions at $T=T_{g}+23.4^{\circ} \mathrm{C}$ after $W i_{R}>1\left(W i_{R}=7.44,7.76,3.34,4.11,1.48,1.77\right.$ for $5 \%, 10 \%, 20 \%, 30 \%, 40 \%$ and $50 \%$ of PS820k, respectively)

As discussed in the Introduction, this unexpectedly high steady viscosity found when diluting the sample has already been observed ${ }^{7}$, and has been discussed by Ianniruberto et $a l .{ }^{15}$ based on the concept of monomeric friction reduction: As one increases the concentration, the stretched and long chains have a lower probability to be surrounded by the nearly isotropic short chains and thus have a higher potential for reduction of monomeric friction. As a consequence, the retraction time decreases and the shrinkage of the chain increases. Thus, according to this concept, polymer chains are stretching more when they are diluted in oligomers.

This picture can also be tested for the transient response. Figure 9 a) shows a comparison of the stress growth coefficient of the samples containing $5 \mathrm{wt} \%, 10 \mathrm{wt} \%$ and $20 \mathrm{wt} \%$ of PS 820 in OS9, deformed at a similar elongation rate. The data have been divided by $\phi$, in order to account for the influence of concentration on the chain density. It is observed that the curves superimpose well, before and during a large part of their strain hardening. Only at long times, 
just before reaching the steady state regime, differences between the transient viscosities of the samples are observed, leading to the $\sqrt{\phi}$ scaling (see Figure $9 \mathrm{~b}$ )). Thus, these results suggest that, at short times, the chains stretch in a similar way in the three samples, with no influence of the concentration and of their molecular environment. According to the friction reduction concept, this means that at these times $(t<150 \mathrm{~s})$, despite the fact that the coalignment of the chains is large enough to induce chain stretch, it stays too low to induce friction reduction. Differences with concentration due to friction reduction only emerge at longer times, when the chain coalignment is more important.

As it has been discussed in refs. [52-54], a different concentration dependence of the steady viscosity could also be due to the emergence of local hydrodynamics when diluting the polymer chains, with the oligomers acting more and more like a good solvent. However, as it is shown in ref. [26], the same steady viscosity is found if oligomers are replaced by short entangled chains (PS chains of $73 \mathrm{~kg} / \mathrm{mol}$ ). The role of the environment is therefore not clear and should be further investigated.

Another possible explanation for the observed $\sqrt{\phi}$ scaling is a different balance between the chain stretching and the way the obstacles (entanglements) are continuously removed by the flow. ${ }^{4}$ As described in ref.[4], this last process occurs proportionally to $\dot{\epsilon}$. However, its efficiency could depend on the sample composition. If we assume that the survival probability of an entanglement depends on the ability of the entanglement segments to deform with the flow, i.e. on the number of Kuhn segments they contain, larger value of $N_{e}$ should allow the chains to stretch more, and consequently the contribution of a chain to the steady viscosity should increase when diluting the polymer. 

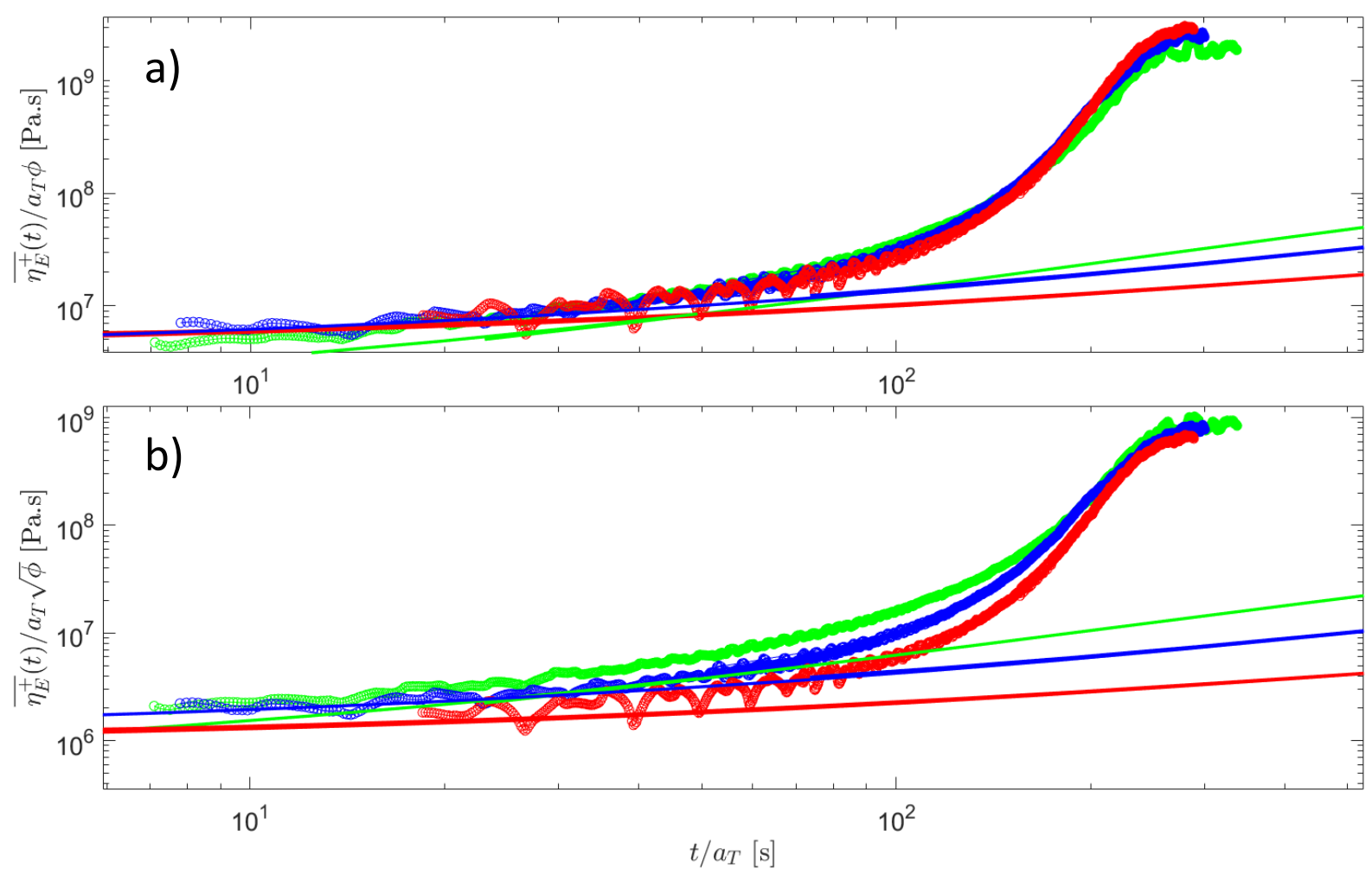

Figure 9: Stress growth coefficient for diluted solutions containing 5 wt\% (red), $10 w t \%$ (blue) or $20 w t \%$ (green) PS820, multiplied by $\phi$ (a) or $\sqrt{\phi}$ (b). Data have been subsequently shifted to $T=T_{g}+23.4^{\circ} \mathrm{C}$. Their corresponding strain rates are 0.0151/s, 0.0142/s and 0.0122/s, respectively.

\section{Intermediate strain rates:}

As shown in Figure 6, at intermediate strain rates another regime, which acts as a transition between the linear and the high stretch regime, can be observed. As the concentration has a much stronger influence on the steady state elongational viscosity in the linear regime than in the high stretch regime, this results in an upturn of the steady state viscosity in function of the elongation rate. Accordingly, the upturn is not observed for highly concentrated solutions, where the zero-elongation viscosity is high.

The upturn is a nonlinear phenomenon due to chain stretch, possibly mitigated by friction reduction effects. With increasing stretch rate, friction reduction is expected to become more and more significant, thus inducing thinning. Furthermore, we conclude that the steady state 
viscosity reached in the non-linear regime should be interpreted independently from the LVE envelope or the transient hardening. These results also demonstrate the ambiguity that arises when the zero-elongation rate viscosity is utilized to normalize the viscoelastic response under large flows, as it has been discussed in ref. [26]. Similar arguments have also been made by O'Connor et al. in ref. [55] in case of polymer melts, to explain the rate dependence of the steady viscosity with varying molar mass and number of Kuhn segments per entanglement segment.

\section{III.3. Elongation viscosity: Influence of the molar mass}

The influence of the molar mass of the long PS chains in solution is studied by looking at samples with different molar mass while keeping a constant level of dilution. Similar to the results introduced in the preceding section, the onset of the steady state values was reached for all strain rates presented in Figure 10. However, for the blends PS261k (20 wt\%) - 9k and PS389k (20 wt \%) - 9k, when the steady state was about to be reached, large oscillations were recorded by the FSR. Despite this, we decided to keep the data as they still allow discussions. From Figure 10, one can observe that the smaller the molar mass of the long chains, the smaller the strain hardening and the greater the ratio between the steady state values and the zeroelongation viscosity $3 \eta_{0}$ becomes. Again, this result seems to be related to the different molecular weight dependence in the linear regime and in the non-linear regime. 

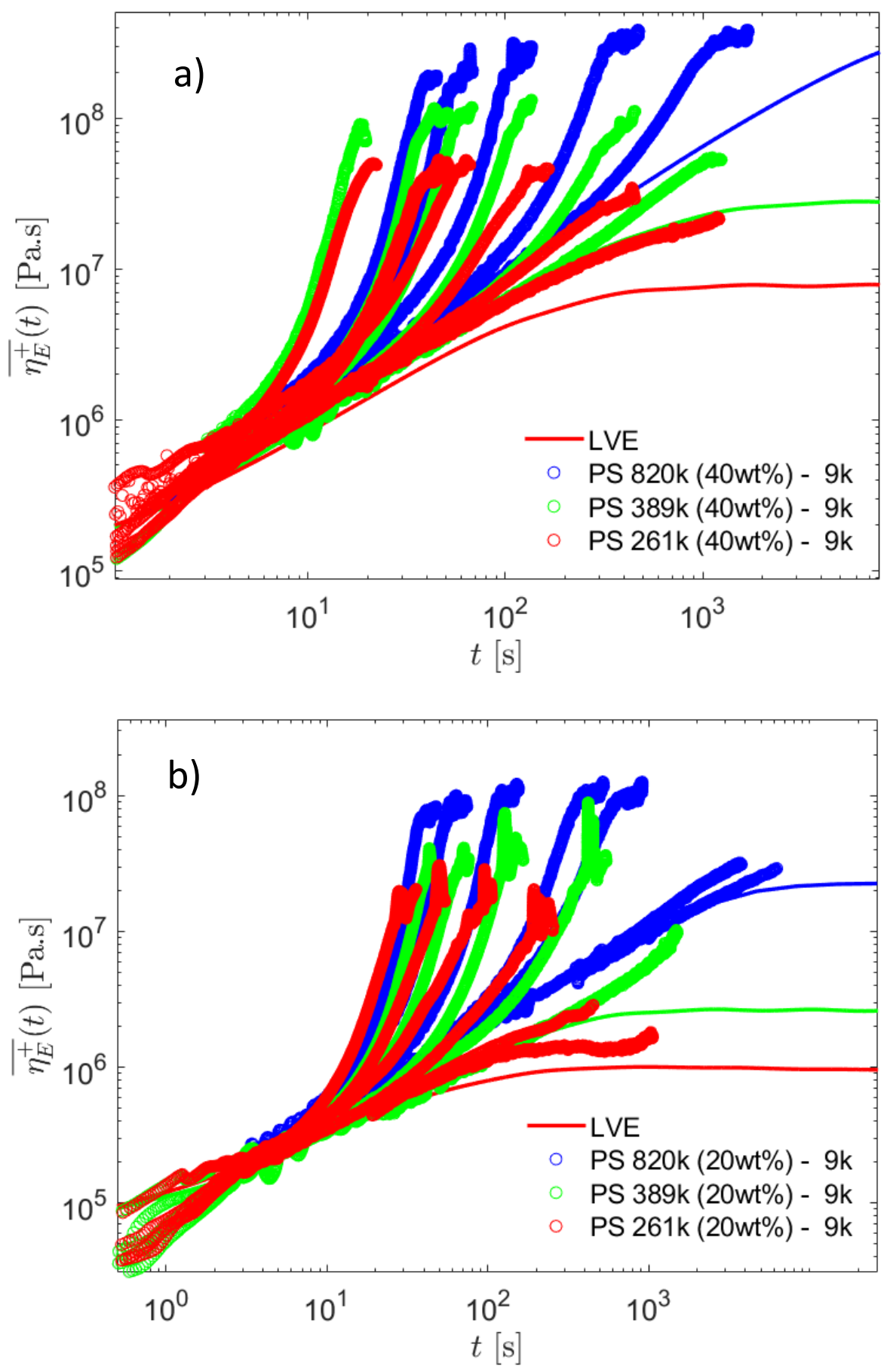

Figure 10: Stress growth coefficient of PS solutions for different strain rates at $T=130^{\circ} \mathrm{C}$. Data were measured for different strain rates. For Figure 10a), the strain rates are from right to left 0.003, 0.01, 0.03, 0.06, 0.09, 0.2 $s^{-1}$ for PS 389k (40wt\%) and PS 261k (40wt\%) and 0.003, 0.01, 0.03, 0.06, $0.09 \mathrm{~s}^{-1}$ for PS $820 k(40 w t \%)-9 k$. For Figure 10b), the strain rates are from right to left 0.003, 0.01, 0.03, 0.06, 0.09 for PS 389k (20 wt\%) and PS $820 \mathrm{k}(20 \mathrm{wt} \%)$ and $0.003,0.01,0.03,0.06,0.09,0.2 \mathrm{~s}^{-1}$ for PS 261k (20wt\%) - 9k. Additionally, for PS 820k (20 wt\%), LVE envelopes have also been measured in elongational flow at 0.0001 and $0.00003 \mathrm{~s}^{-1}$ respectively. All the data have been subsequently shifted to $T=T_{g}+23.4{ }^{\circ} \mathrm{C}$. 
This effect can be seen better in Figure 11, which plots the steady state viscosity of the solutions as a function of the strain rates. Indeed, the linear regime is much more affected by the molar mass of the chains than the non-linear regime.

The curves also show a similar three-regime picture as described in the previous section. In the linear regime, the steady state viscosity scales with $\eta_{E} \propto M_{w}^{x}$, where $x \in[3,3.4]$, while, in the non-linear regime, as shown in Figure 12, it scales linearly with the molecular mass of the chains. At intermediate strain rates, there is an upturn of the steady state viscosity, which becomes much more prominent for the low molar mass samples.

The linear scaling in the high stretch regime is in discrepancy to the quadratic scaling predicted by Equation 9 for the case that all chains are oriented and stretched nearly to full extension. The linear scaling extends the same observation made for melts by Bach et al. ${ }^{5}$ now also to polymer solutions. It indicates that the chains are not reaching their finite extensibility, and that the stretch state of the chains in the steady regime is only governed by their Rouse time. 


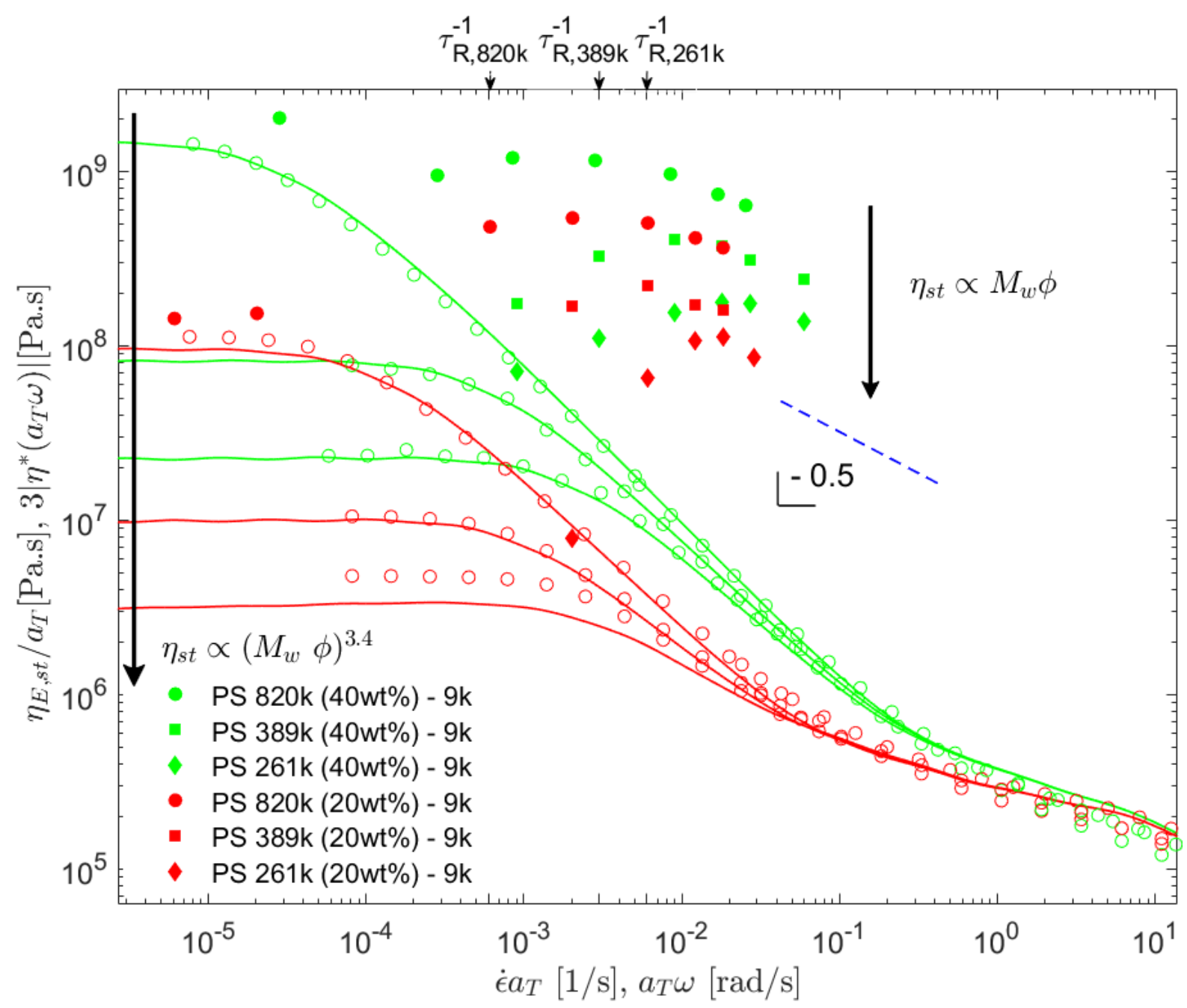

Figure 11: Elongational steady state viscosity (closed symbols) of PS solutions as a function of the strain rate. The corresponding LVE data (open symbols) are also shown. The solid lines represent the predictions of TMA tube-based model ${ }^{40}$. All data are shifted to $T=T_{g}+23.4{ }^{\circ} \mathrm{C}$. 

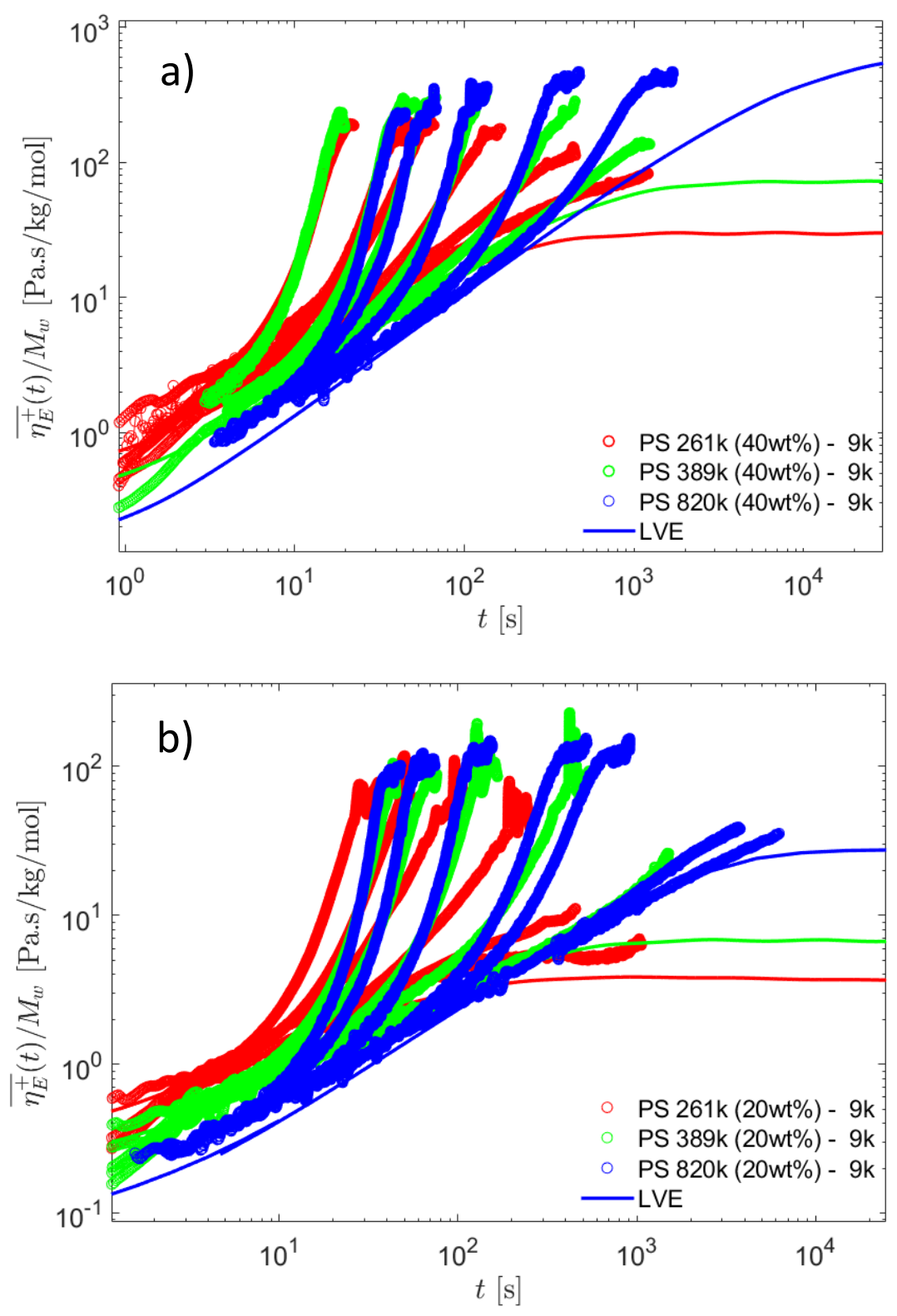

Figure 12: Stress growth coefficient $\eta_{E}^{+}$of PS solutions divided by the molar mass.

\section{III.4. Elongation viscosity: Influence of the polydispersity}

In this section, we study the response of bidisperse systems of long and short chains. The bidisperse blends are used as model systems to investigate the influence of the polydispersity on the extensional viscosity of PS systems.

The short components in the bimodal blends are PS256, PS261 and PS389. They have been selected so that the highest strain rates exceed significantly their reciprocal Rouse time $\tau_{R}^{-1}$, 
ensuring that they are also stretched (see Table I). It should be noticed, however, that this criterion may not be valid if the concept of reduction of the monomeric friction is considered. Indeed, in such a case the initial stretching of the long chains is expected to make the environment of the short chains more anisotropic and thus to delay the onset of their stretching. However, we believe that the strain rates used here are high enough to compensate any possible decrease of the stretch relaxation time.

In addition to ensuring chain stretching, the molar mass of the chains is selected sufficiently high to prevent the effect of tube dilation. Indeed, the Graessley number $G r=\frac{M_{L} M_{e}^{2}}{M_{S}^{3}}$ of the systems is much higher than the critical Graessley number $G r_{c}{ }^{56}$. Therefore, the entanglements with short chains are long-lasting and the long chains relax in a thin tube formed by entanglements with both long and short chains. This means that all the systems have the same finite extensibility $\lambda_{\max }=\sqrt{N_{e}}$, and each entanglement segment has the same ability to stretch. As a consequence, in absence of constraint release, the steady state viscosity at high Weissenberg numbers should be given by $\eta_{E, s t}=\sum_{i} \phi_{i} \eta_{E, m e l t, i}$ according to the DEMG model $^{3}$, with $\eta_{\text {melt }, i}$ referring to the steady state viscosity of monodisperse melts, which have chains with same length as in the bimodal systems. Indeed, in the preceding sections we have shown that the steady state extensional viscosity of long chains diluted in unentangled short chains scales with $M_{w} \sqrt{\phi}$, and we attributed the $\sqrt{\phi}$ scaling to the different extensibility of the entanglement blobs. This scaling is therefore expected to disappear here for bidisperse blends, in which both components are entangled. Similarly, according to the concept of friction reduction, viscosity is expected to approximately scale with $M_{w} \phi$, since changing the concentration in bidisperse blends should not modify significantly the anisotropic character of the environment, contrary to solutions, in which the overall fraction of the stretched chains evolves with concentration, thus affecting the anisotropy of the environment at the Kuhn 
segment scale. Therefore, these blends should have similar potential for the reduction of monomeric friction.

The stress growth coefficient $\eta_{E}^{+}(t)$ is shown in Figure 13 for three bidisperse systems. Steady states have been reached for the high strain rates as shown in Figure S3 of the Supplemental Material (SM). At intermediate rates, however, the steady regime is not observed.

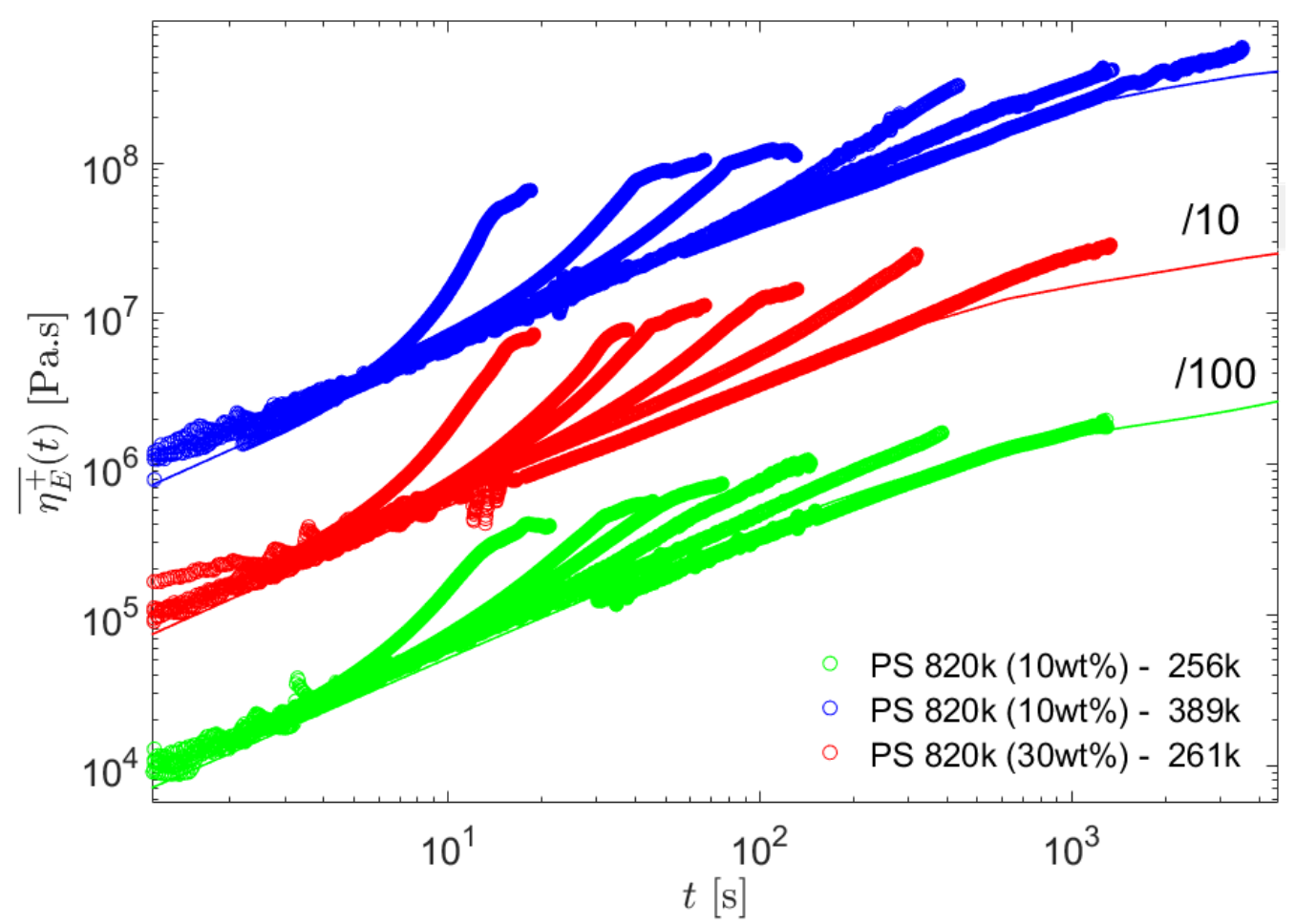

Figure 13: Stress growth coefficient $\eta_{E}^{+}(t)$ of bidisperse blends. Data were measured for different strain rates: 0.003, 0.01, 0.03, 0.06, 0.09, $0.2 \mathrm{~s}^{-1}$ at $\mathrm{T}=138^{\circ} \mathrm{C}$ ( or $\mathrm{T}-T_{g}=31.4^{\circ} \mathrm{C}$ ). For clarity, some of the extensional data has been downshifted by 1 or 2 decades.

In order to determine the steady viscosity of a polymer blend, the two mixing laws discussed above are tested. The first one is based on the scaling found for polymer solutions, $\eta_{E} \propto M_{w} \sqrt{\phi}$ ( $\phi$ represents the weight fraction of polymer chains diluted in oligomers). If this scaling is extended to polymer blends, one expects that the contribution of a specific polymer of mass $M_{i}$ and Rouse time $\tau_{R, i}$ to the steady viscosity will be proportional to the square root of its weight 
fraction, $\sqrt{\phi_{i}}$. In this case, one can define an effective Rouse time $\tau_{R}^{\alpha}=\left(\sum_{i} \sqrt{\phi_{i} \tau_{R, i}}\right)^{2}$ for polydisperse systems via:

$$
\begin{aligned}
\eta_{E, S t} & =\sum_{i} \eta_{E, s t, i} \\
& =A G_{N}^{0} \dot{\epsilon}^{-0.5} \sum_{i} \sqrt{\phi_{i} \tau_{R, i}} \\
& =A G_{N}^{0} \dot{\epsilon}^{-0.5} \sqrt{\tau_{R}^{\alpha}}
\end{aligned}
$$

On the other hand, if we follow the second mixing law and assume that $\eta_{E} \propto M_{w} \phi$, an effective Rouse time $\tau_{R}^{\beta}$ for polydisperse systems can be expressed as follows.

$$
\tau_{R}^{\beta}=\left(\sum_{i} \phi_{i} \sqrt{\tau_{R, i}}\right)^{2}
$$

In Figure 14, we evaluated these two relations for the bidisperse data. The blue line comes from the linear regression shown in Figure 7. It is observed that the data superimpose very well if a scaling $\eta_{E} \propto \sum_{i} \phi_{i} \sqrt{\tau_{R, i}}$ is considered, and that the normalized viscosity of all the bidisperse blends is found to follow a similar behavior. Thus, these results suggest that a blending rule, as the one proposed in Equation 13, can be determined to explain the steady viscosity of the entangled bidisperse polymers. However, it is valid only if the chains stay well entangled. Furthermore, these results are in agreement with both the friction reduction concept and the idea that the proportion of the time at which the transient viscosity reaches its steady regime depends on the stretchability of entanglement blobs. 


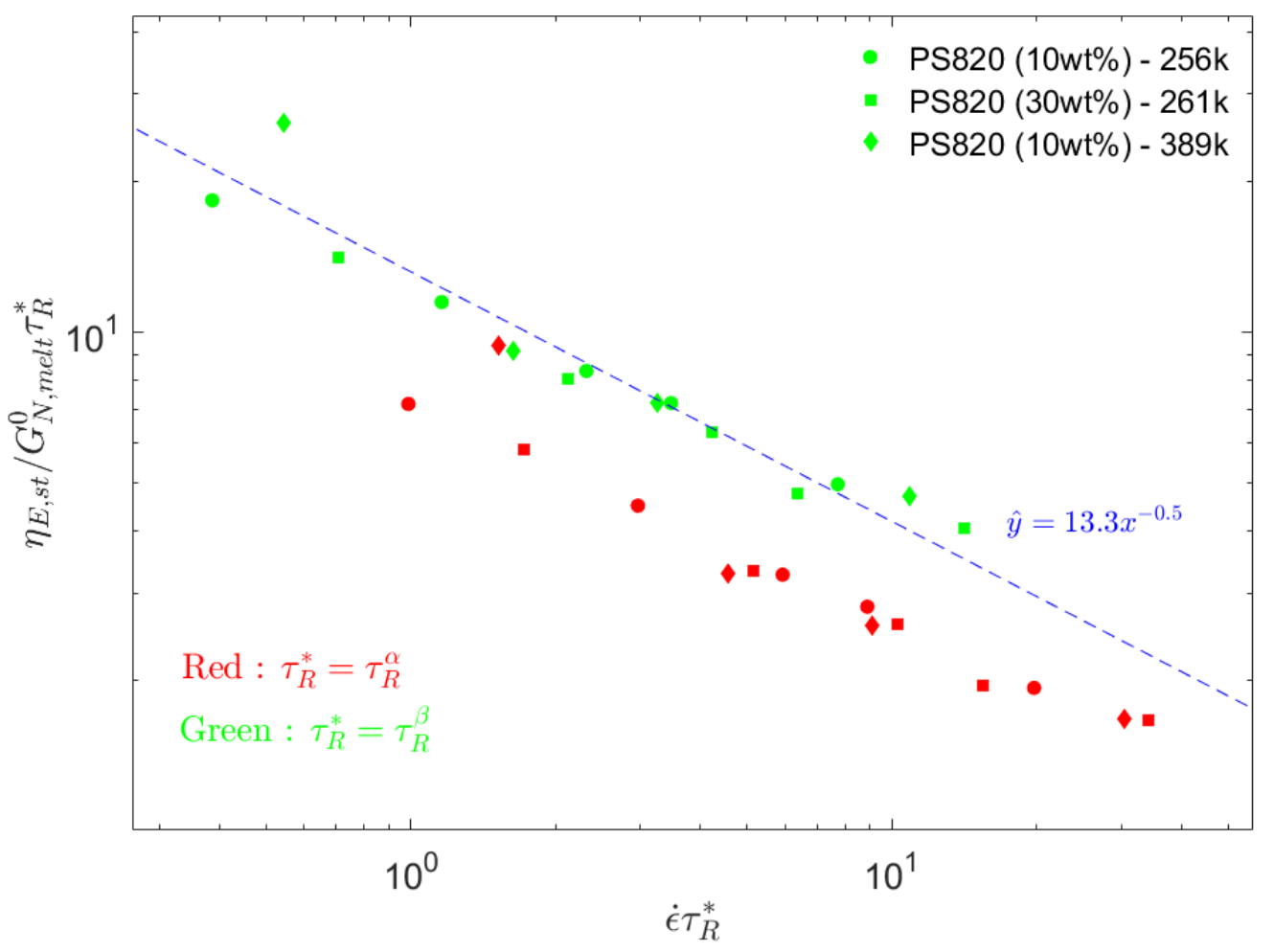

Figure 14: Assessment of the empirical law (see text) for bidisperse blends

In order to validate the $\eta_{E, s t}=\sum_{i} \phi_{i} \eta_{E, \text { melt }, i}$ scaling law, the stress growth coefficient of the two bimodal systems with very similar average molar mass $\overline{M_{w}}=\sum_{i} \phi_{i} M_{w, i}$ and containing well entangled short chains has been measured. As observed in Figure 15, the steady state viscosities of the samples almost superimpose at high strain rates. 


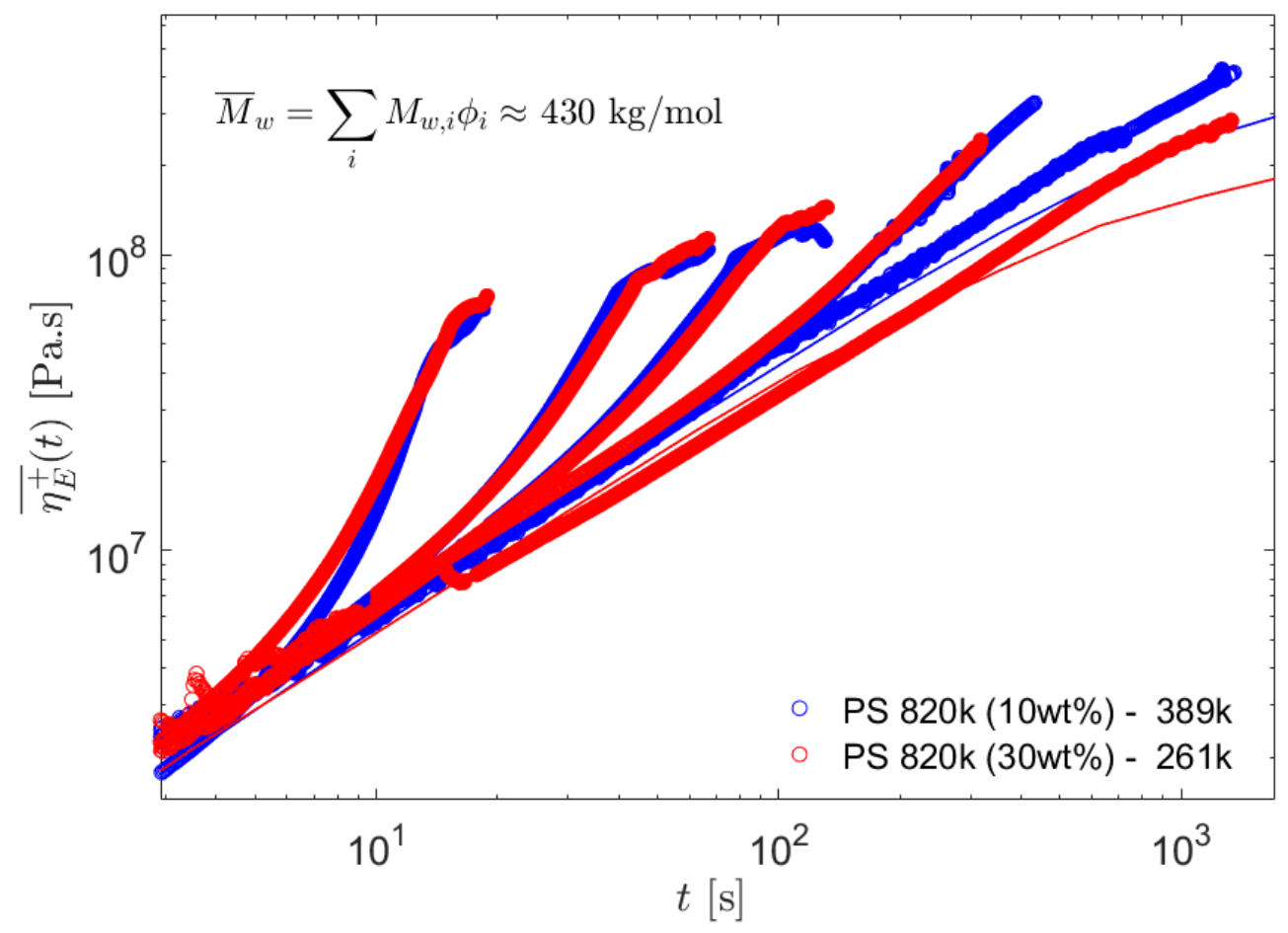

Figure 15: Stress growth coefficient of two bimodal systems which have very similar average molar mass $\overline{M_{w}}$ (i.e.

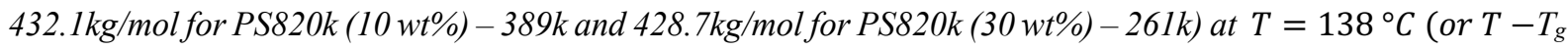
$\left.=31.4^{\circ} \mathrm{C}\right)$.

One can also see that the transient responses of the systems are similar. Thus, this confirms the proposed $\phi$ scaling of the steady viscosity in the well-entangled regime, while a $\sqrt{\phi}$ scaling is observed in solution.

\section{III.5. Elongation steady state viscosity: Overall scaling for polymer melts and solutions}

Based on our current observation, the overall scaling for polymer solutions and bimodal systems, at high strain rates, is respectively $\eta_{E, s t} \propto M_{w} \sqrt{\phi}$ and $\eta_{E, s t} \propto \sum_{i} \phi_{i} M_{w, i}$. It must be noted that in these expressions, $\phi$ is used to describe the volume fraction of the polymer diluted in unentangled short chains or oligomers, while $\phi_{i}$ is used to describe the volume fraction of a component in a bidisperse entangled blend. These two scaling laws can be put together, as it is proposed in Figure 16, which shows the normalized steady state viscosity value of all measured polymer systems as a function of the Weissenberg number $\left(W i_{R}=\dot{\varepsilon} \tau_{R}\right)$. For the bidisperse 
blends, Equation 13 has been used to define the Rouse time $\tau_{R}^{*}$. Additionally, steady state viscosities of two polymer melts coming from ref [7] have been added to the dataset.

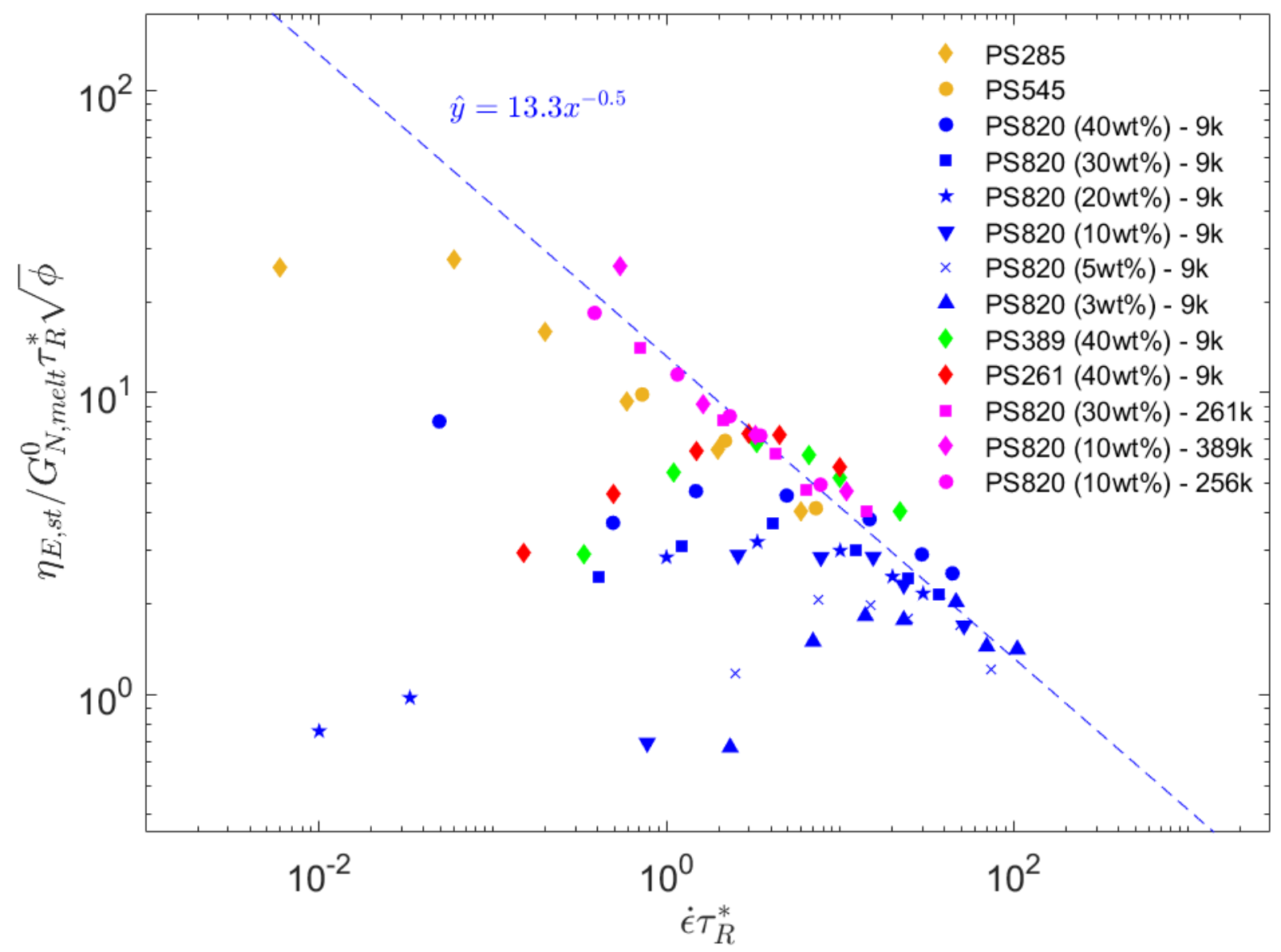

Figure 16: Dimensionless elongational steady state viscosities $\eta_{E, s t} / G_{N, m e l t}^{0} \tau_{R}^{*} \sqrt{\phi}$ of PS systems as a function of the Weissenberg number $W i_{R}=\dot{\varepsilon} \tau_{R}^{*}$. All the data are shifted at $T=T_{g}+23.4^{\circ} \mathrm{C}$.

As expected, at high Weissenberg numbers a universal trend seems to emerges from the data, where all PS systems follow a common slope of $-1 / 2$.

\section{Conclusion}

In this work, we measured and discussed the non-linear extensional responses of moderate and relatively high molecular weight monodisperse PS chains, diluted, or not, with oligomers at 
various concentrations. The concentrations were chosen so that all the polymeric systems stay entangled.

For all measured systems, three distinct regimes of the rate dependent steady state elongational viscosity $\eta_{E, s t}$, with different dependencies on the concentration and molar mass, were observed. At very low strain rates, the polymeric chains succeed to maintain an equilibrium structure and the steady state extensional viscosity tends to the Trouton value $3 \eta_{0}$. At high strain rates, polymer chains are predominantly stretched and exhibit monotonous extension thinning. In this high stretch regime, three main results have been obtained:

1. First, we proved that it is possible to find a scaling law that describes the influence of the concentration for the steady state extensional viscosity $\eta_{E, s t}$ of entangled polymer solutions. In particularly, in this work we demonstrated that the steady viscosity of PS chains dissolved in oligomers of 9k evolves with the square root of the concentration.

2. Secondly, we demonstrated that the linear evolution of the steady state extensional viscosity with the molar mass, that was first observed by Bach et al. ${ }^{5}$ for melts, can be extended to polymeric solutions.

3. Thirdly, the results showed that the steady state extensional viscosity $\eta_{E, s t}$ of bimodal entangled systems follow the simple mixing rule: $\eta_{E, s t} \propto \sum_{i} M_{w i} \phi_{i}$ where $M_{w i}$ and $\phi_{i}$ are the molar mass and the weight fraction of the components of the bimodal blends.

For polymer solutions, we showed that the steady viscosity shows an unexpected dependence on concentration, well described by a $\sqrt{\phi}$ scaling, while the corresponding transient hardening well scales with $\phi$. The $\sqrt{\phi}$ dependence of steady viscosity can be explained in the framework of friction reduction if we assume that only in the steady regime the chain stretch is large enough to lead to friction reduction. This scaling could also be explained if we assume that the flow- 
induced disentanglement of a chain depends on the ability of its entanglement segments to deform with the flow, i.e. on the number of Kuhn segments they contain.

In the high stretch regime, we observe that the importance of the transient strain hardening strongly depends on the sample concentration, becoming very large when diluting the polymer chains in oligomers. This was attributed to the different concentration and molar mass dependence of the linear envelop and of the steady state viscosity under strong elongational flow. As strain hardening occurs in the rubbery plateau for the last experimentally reachable strain rate regime for entangled polymeric solutions, it scales with $\frac{\eta_{E, s t}(\dot{\epsilon})}{\eta_{L V E}} \propto \frac{M_{w} \sqrt{\phi}}{\phi^{2} G_{N}^{0}} \frac{\dot{\epsilon}}{\sqrt{\dot{\epsilon}}} \propto \frac{M_{w}}{\phi^{3 / 2}} \sqrt{\dot{\epsilon}}$. This explains the well-known result that the strain hardening tends to increase when increasing the molar mass or decreasing the concentration. Furthermore, the ratio of the steady state extensional viscosity to the zero-elongation viscosity scales with $\frac{\eta_{E, s t}}{3 \eta_{0}}=\frac{M_{w} \sqrt{\phi}}{\left(M_{w} \phi\right)^{x}} \frac{1}{\sqrt{\dot{\epsilon}}}=$ $\phi^{\frac{1}{2}-x} M_{w}^{1-x} \frac{1}{\sqrt{\grave{\epsilon}}}$, where $x \in[3,3.4]$. As a consequence, increasing the molar mass and/or the concentration leads to an increase of the amount of steady thinning. From this scaling, it is also seen that a similar strain hardening behavior cannot be obtained if a larger dilution of the polymer is compensated by a higher molar mass of the chains, in such a way that $M_{w} \phi$ is constant.

Due to these different scaling laws, at intermediate strain rates, another regime is observed, which acts as a transition between the linear and the non-linear regime. As the dependence on the concentration is much higher in the linear regime than in its non-linear counterpart, this results in an upturn of the steady state elongational viscosity with rate at the transition between these two regimes. Accordingly, this upturn is missing for highly concentrated solutions and high molar masses, where the zero-elongation viscosity is high. 
Finally, based on our results, we proposed a scaling law to determine the steady viscosities of polymer blends and solutions, which allowed us to build a universal steady viscosity curve in the stretch regime.

\section{Acknowledgments}

The authors thank Prof. Dimitris Vlassopoulos and Q. Huang for very helpful discussions. This work was funded by DSM Materials Science Center, and by the French Community of Belgium through ARC project N¹6/21-076. E.V.R. is Research Associate of the FRS-FNRS. 


\section{Appendix}

In this work, several polymeric systems have been compared at different temperatures, under iso- $T_{g}$ condition. Accordingly, the shear and elongational experimental data have been shifted using TTS shift factors which can be validated by the overlapping with the WLF curve of PS melts. In this appendix, we confirm that TTS shift factors are also applicable in the non-linear elongational regime by testing a sample at different temperature and corresponding rates.

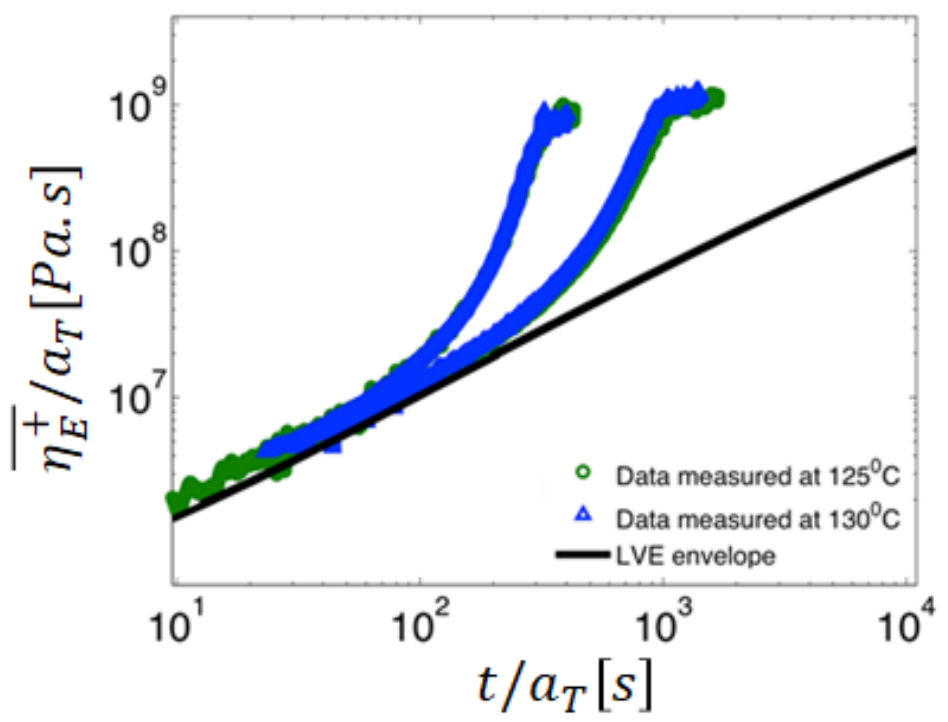

Figure 17: Validating TTS shift factors in the non-linear flow. Green symbol represents data measured at $125^{\circ} \mathrm{C}$ at 0.003 and $0.01 \mathrm{~s}^{-1}$ and blue symbol represent data measured at $130^{\circ} \mathrm{C}$ at 0.01 and $0.03 \mathrm{~s}^{-1}$.

As shown in Figure 17, the solutions PS820 (40 wt\%) was measured at two different strain rates 0.003 and $0.01 \mathrm{~s}^{-1}$ at $125^{\circ} \mathrm{C}$ (i.e. at the same iso- $T_{g}$ condition used in this work). With TTS shift factor of 3.86 (from 125 to $130{ }^{\circ} \mathrm{C}$ ), these strain rates correspond approximately to 0.01 and $0.03 \mathrm{~s}^{-1}$ at $130^{\circ} \mathrm{C}$. As observed, these shifted data coincide with data measured at $130{ }^{\circ} \mathrm{C}$ at corresponding rates, thus ensuring that TTS principle works well in the non-linear regime. 
Supporting Information: Measured extensional stress as a function of Hencky strain of the different samples.

\section{References}

[1] M. Doi, S. F. Edwards, The Theory of Polymer Dynamics; Clarendon Press: Oxford, (1986).

[2] A.E. Likhtman and T.C.B. McLeish, Quantitative theory for linear dynamics of linear entangled polymers. Macromolecules 35, 6332, (2002).

[3] J. M. Dealy, R. G. Larson, Structures and Rheology of Molten Polymers, 2nd Edition, Carl Hanser Verlag GmbH \& Co., (2018).

[4] G. Marrucci, G. Ianniruberto, Interchain pressure effect in extensional flows of entangled polymer melts. Macromolecules, 37, 3934-3942, (2004).

[5] A. Bach, K. Almdal, H. K. Rasmussen, O. Hassager, Elongational viscosity of narrow molar mass distribution polystyrene. Macromolecules 36, 5174-5179, (2003).

[6] P. K. Bhattacharjee, , J. P. Oberhauser, , G. H. McKinley, L. G. Leal, T. Sridhar, Extensional rheometry of entangled solutions. Macromolecules 35, 10131-10148, (2002).

[7] Q. Huang, O. Mednova, H. K. Rasmussen, N. J. Alvarez, A. L. Skov, K. Almdal, O. Hassager, Concentrated polymer solutions are different from melts: Role of entanglement molecular weight. Macromolecules 46, 5026-5035, (2013).

[8] S. Q. Wang, Nonlinear Polymer Rheology: Macroscopic phenomenology and Molecular foundation, Wiley: Hoboken, NJ, (2017).

[9] M. H. Wagner, V. H. Rolón-Garrido, Nonlinear rheology of linear polymer melts: modeling chains stratch by interchain tube pressure and Rouse time. Korea-Australia Rheol. J., 21, 203211, (2009).

[10] M. H. Wagner, An extended interchain tube pressure model for elongational flow of polystyrene melts and concentrated solutions. J. Non-Newtonian Fluid Mech, 222, 121-131, (2015).

[11] M. H. Wagner, E. Narimissa, V. H. Rolón-Garrido, From melt to solution: Scaling relations for concentrated polystyrene solutions. Journal of Rheology, 59, 1113-1130, (2015).

[12] M.H. Wagner, Scaling relations for elongational flow of polystyrene melts and concentrated solutions of polystyrene in oligomeric styrene. Rheol Acta, 53, 765-777, (2014).

[13] E. Van Ruymbeke, J. Nielsen, O. Hassager, Linear and nonlinear viscoelastic properties of bidisperse linear polymers : Mixing law and tube pressure effect. Journal of Rheology, 54, 1155, (2010). 
[14] E. Narimissa, Q. Huang, M. H. Wagner, Elongational rheology of polystyrene melts and solutions: Concentration dependence of the interchain tube pressure effect. Journal of Rheology, 64(1), 95-110, (2020).

[15] G. Ianniruberto, A. Brasiello, G. Marrucci, Friction coefficient does not stay constant in non linear viscoelasticity, Proc. 7th Annu., Eur. Rheol. Onf., 1, 61, (2011).

[16] T. Yaoita, T. Isaki, Y. Masubuchi, H. Watanabe, G. Ianniruberto, G. Marrucci, Primitive chain network simulation of elongational flows of entangled linear chains: stretch/orientationinduced reduction of monomeric friction. Macromolecules, 45, 2773-2782, (2012).

[17] G. Ianniruberto, G. Marrucci and Y. Masubuchi, "Melts of Linear Polymers in Fast Flows", Macromolecules 53, 5023-5033 (2020).

[18] Y. Matsumiya, H. Watanabe, "Non-Universal Features in Uniaxially Extensional Rheology of Linear Polymer Melts and Concentrated Solutions: A Review", Progress in Polymer Science 112, 101325 (2021).

[19] G. Ianniruberto, A. Brasiello, and G. Marrucci, Simulations of fast shear flows of PS oligomers confirm monomeric friction reduction in fast elongational flows of monodisperse PS melts as indicated byheooptical data. Macromolecules, 45, 8058-8066, (2012).

[20] Y. Masubuchi, T. Yaoita, Y. Matsumiya, H. Watanabe, G. Ianniruberto, and G. Marrucci, Stretch/orientation induced acceleration in stress relaxation in coarse-grained molecular dynamics simulations. Nihon Reoroji Gakkaishi, 41(1), 35-37, (2013).

[21] P. S. Desai and R. G. Larson, Constitutive model that shows extension thickening for entangled solutions and extension thinning for melts. J. Rheol., 58, 255-279, (2014).

[22] Q. Huang, N. J. Alvarez, Y. Matsumiya, H. K. Rasmussen, H. Watanabe, and O. Hassager, Extensional rheology of entangled polystyrene solutions suggest importance of nematic interactions. ACS Macro Lett. 2, 741-744, (2013a).

[23] G. Ianniruberto, Extensional flows of solutions of entangled polymers confirm reduction of friction coefficient. Macromolecules 48, 6306-6312, (2015).

[24] Q. Huang, L. Hengeller, N. J. Alvarez, O. Hassager, Bridging the Gap between Polymer Melts and Solutions in Extensional Rheology. Macromolecules, 48, 12, 4158-4163, (2015).

[25] G. W. Park, G. Ianniruberto, Flow-induced nematic interaction and friction reduction successfully describe PS melt and solution data in extension startup and relaxation. Macromolecules, 50, 4787-4796, (2017).

[26] T. Shahid, C. Clasen, F. Oosterlinck, E. van Ruymbeke, Diluting Entangled Polymers Affects Transient Hardening but Not Their Steady Elongational Viscosity. Macromolecules, $52,6,2521-2530,(2019)$.

[27] Y. Matsumiya, H. Watanabe, Y. Masubuchi, Q. Huang, O. Hassager, Nonlinear Elongational Rheology of Unentangled Polystyrene and Poly (p-tert-butylstyrene) Melts. Macromolecules, 51, 9710-9729, (2018). 
[28] G. Ianniruberto, A. Brasiello, G. Marrucci, Modeling Unentangled Polystyrene Melts in Fast Elongational Flows. Macromolecules, 52, 4610-4616, (2019).

[29] S. V. Bobbili and S. T. Milner, "Chain tension reduces monomer friction in simulated polymer melts”, J.Rheol. 64, 1373 (2020).

[30] R. H. Colby, D.C. Boris, W.E. Krause, S. Dou, "Shear thinning of unentangled flexible polymer liquids, Rheol. Acta 46(5), 569-575 (2007).

[31] T. C. O'Connor, A. Hopkins, M. O. Robbins, Stress Relaxation in Highly Oriented Melts of Entangled Polymers. Macromolecules, 52, 22, 8540-8550, (2019).

[32] G. Ianniruberto, G. Marrucci, Molecular Dynamics Reveals a Dramatic Drop of the Friction Coefficient in Fast Flows of Polymer Melts. Macromolecules, 53 (7), 2627-2633, (2020).

[33] T. Shahid., Q. Huang, F. Oosterlinck, C. Clasen, E. van Ruymbeke, Dynamic dilution exponent in monodisperse entangled polymer solutions. Soft Matter, 13(1), 269-282, (2017).

[34] T. G. Fox, Influence of Diluent and of Copolymer Composition on the Glass Temperature of a Poly-mer System. Bulletin of the American Physical Society, 1, 123, (1956).

[35] M. L. Williams, R. F. Landel, and J. D. Ferry, The Temperature Dependence of Relaxation Mechanisms in Amorphous Polymers and Other Glass-forming Liquids. J. Am. Chern. Soc., 77, 3701, (1955).

[36] C.-Y. Liu, J. He, R. Keunings, C. Bailly, New Linearized Relation for the Universal Viscosity-Temperature Behavior of Polymer Melts. Macromolecules, 39(25), 8867-8869, (2006).

[37] J. M. R. Marin, J. K. Huusom, , N. J Alvarez, Q. Huang, H. K. Rasmussen, A. Bach, A. L. Skov, O. Hassager, A control scheme for filament stretching rheometers with application to polymer melts. J. Non-Newtonian Fluid Mech, 194, 14-22, (2013).

[38] H. K. Rasmussen, A.G. Bejenariu, O. Hassager, D. Auhl, Experimental evaluation of the pure configurational stress assumption in the flow dynamics of entangled polymer melts. J. Rheol, 54(6), 1325-1336, (2010).

[39] I. M. Kolte, H. K. Rasmussen, O. Hassager, Transient filament stretching rheometer II : Numerical simulation. Rheol. Acta, 36, 285-302, (1997).

[40] E. Van Ruymbeke, R. Keunings, C. Bailly, Prediction of linear viscoelastic properties for polydisperse mixtures of entangled star and linear polymers: Modified tube-based model and comparison with experimental results. Journal of Non-Newtonian Fluid Mechanics, 128(1), 722, (2005).

[41] S. L. Morelly, L. Palmese, H. Watanabe, N. J. Alvarez, Effect of Finite Extensibility on Nonlinear Extensional Rheology of Polymer Melts. Macromolecules, 52, 3, 915-922 , (2019). 
[42] E. Van Ruymbeke, Y. Masubuchi, H. Watanabe, Effective Value of the Dynamic Dilution Exponent in Bidisperse Linear Polymers: From 1 to 4/3. Macromolecules, 45, 4, 2085-2098, (2012).

[43] C.R Lopez Barron, Y. Zeng, J. J. Richards, Chain Stretching and recoiling during startup and cessation of extensional flow of bidisperse polystyrene blends. Journal of Rheology, 61(4), 697-710, (2017).

[44] D. Auhl, P. Chambon, T McLeish, D. Read, Elongational Flow of Blends of Long and Short Polymers, Effective Stretch Relaxation Time. Physical Review Letters, 103(13), 136001, (2009).

[45] G. Marrucci, Relaxation by reptation and tube enlargement: A model for polydisperse polymers. Journal of Polymer Science: Polymer Physics Edition, 23(1), 159-177, (1985).

[46] Q. Huang, S. Agostini, L. Hengeller, M. Shivokhin, N. J. Alvarez, L. R. Hutchings, O. Hassager, Dynamics of Star Polymers in Fast Extensional Flow and Stress Relaxation. Macromolecules, 49(17), 6694-6699, (2016).

[47] Q. Huang, J. Ahn, D. Parisi, T. Chang, O. Hassager, S. Panyukov, M. Rubinstein, D. Vlassopoulos, Unexpected Stretching of Entangled Ring Macromolecules. Physical Review Letters, 122(20), (2019).

[48] T. Sridhar, M. Acharya, D. A. Nguyen, P. K. Bhattacharjee, On the Extensional Rheology of Polymer Melts and Concentrated Solutions. Macromolecules, 47(1), 379-386, (2013).

[49] N. J. Alvarez, J. M. R. Marín, Q. Huang, M. L. Michelsen, O. Hassager, Creep Measurements Confirm Steady Flow after Stress Maximum in Extension of Branched Polymer Melts. Physical Review Letters, 110(16), (2013).

[50] Q. Huang, S. Costanzo, C. Das, D. Vlassopoulos, Stress growth and relaxation of dendritically branched macromolecules in shear and uniaxial extension. Journal of Rheology, 61(1), 35-47, (2017).

[51] O. Hassager, Kinetic Theory and Rheology of Bead-rod Models for Macromolecular Solutions. I. Equilibrium and Steady Flow Properties. J. Chem. Phys. 1974, 60 (5), 2111-2124.

[52] C.M. Schroeder, "Single polymer dynamics for molecular rheology", Journal of Rheology 62, 371-403, (2018).

[53] C.D. Young, C. E. Sing, "Simulation of semidilute polymer solutions in planar extensional via conformationally averaged Brownian noise”, J. Chem. Phys. 151, 124907, (2019).

[54] C. D. Young, Y. Zhou, C.M. Schroeder, C. E. Sing, "Dynamics and Rheology of RingLinear Blend Semidilute Solutions in Extensional Flow: Modeling and Molecular Simulations", accepted, arXiv:2011.01386v2 [cond-mat.soft] 7 Jan, (2021).

[55] O’Connor T. C., N. J. Alvarez and M. O. Robbins, "Relating chain conformations to extensional stress in entangled polymer melts", Physical review letters 121, 047801, (2018).

[56] S. J. Park, R. G. Larson Tube Dilation and Reptation in Binary Blends of Monodisperse Linear Polymers. Macromolecules, 37(2), 597-604, (2004). 